\title{
BOSQUESCUELA. UN MODELO DE ESCUELA SOSTENIBLE EN LA NATURALEZA
}

\section{Bosquescuela. A model for sustainable learning in nature}

\author{
Philip BRUCHNeR \\ Centro Bosquescuela Cerceda, Cerrillo de Agustín, s/n \\ 284I2 Cerceda (Madrid) \\ Correo-e: philip@bosquescuela.com \\ Antonio Aragón Rebollo \\ Centro Bosquescuela Cerceda, Cerrillo de Agustín, s/n \\ 28412 Cerceda (Madrid) \\ Correo-e: toni.aragon@patrimonionatural.org \\ Recibido: 3I de octubre de 2019 \\ Envío a informantes: 19 de noviembre de 2019 \\ Aceptación definitiva: 28 de noviembre de 2020
}

RESUMEN: Bosquescuela es un modelo educativo que desarrolla el currículum al aire libre. Las distintas propuestas pedagógicas se desarrollan en el exterior: bosque, campo, playa, parques, zonas urbanas. Como entorno principal de aprendizaje se considera la naturaleza. Este modelo se basa en dos pilares: I. El entorno natural ofrece un marco completo que cubre las necesidades del alumnado de un desarrollo global y equilibrado y 2. La curiosidad es uno de los motores principales del aprendizaje. La organización de las clases diarias incluye momentos para hacer hincapié en el desarrollo psicomotor, cognitivo y emocional de los niños y niñas mediante excursiones en las que corren, trepan, conocen el medio ambiente, y clases diarias de iniciación a la lectoescritura y las matemáticas utilizando elementos del entorno como piedras, hojas, ramas, etc. Otra fase protagonista diaria es de juego espontáneo y libre que fomenta el sentido de exploración, el trabajo en equipo y ofrece momentos de alta concentración. La única instalación que se requiere es una cabaña que cumple la función de refugio. Por último, el modelo Bosquescuela es un ejemplo de escuela sostenible coherente en forma y contenido. Así, pretende introducir de manera vivencial los Objetivos de Desarrollo Sostenible adoptados por la Organización de las Naciones Unidas. De manera directa, contribuye al oDs 4 
sobre educación de calidad, al oDs I3 de acción por el clima como principal reto del siglo XXI para la humanidad, al oDs is que aboga por la vida de los ecosistemas terrestres y al ODS 17 sobre la necesidad de crear alianzas para lograr los objetivos.

Palabras Clave: cambio climático; desarrollo sostenible; educación infantil al aire libre; innovación educativa; educación medioambiental.

Aвstract: Bosquescuela is an educational model where the curriculum is developed in nature and where nature provides the environment for learning. In other words, different pedagogical activities take place outside (forest, countryside, beach, urban areas). The two underlying ideas are that I) The natural environment offers everything children require for an adequate and balanced development. 2) Curiosity is one of the main driving forces for learning. The organisation of the daily classes includes periods of psychomotor development in the form excursions where they can run, climb, get to know the environment, invent games, and get started in reading, writing and mathematics using naturally occurring elements such as stones, sticks, leaves, etc. The only infrastructure that is needed is a cabin to provide shelter. Lastly, it should be noted that the Bosquescuela Project is an example of a sustainable school in both it's physical structure and in it's running. As such it strives to implement the Sustainable Development Goals (sDGs) adopted by the United Nations. Bosquescuela contributes directly to SDG 4 concerning quality education, SDG 13 which considers climate action to be humanity's biggest challenge for the $2 \mathrm{I}^{\mathrm{ST}}$ century, SDG $\mathrm{Is}$ that pledges protection for terrestrial ecosystems and SDG I7 which states the need for creating partnerships to achieve the goals.

KEY WORDS: climate change; sustainable development; outdoor preschool education; educational innovation; environmental awareness.

\section{Historia del modelo}

2 OSQUESCUELA ES UN MODELO de educación infantil al aire libre cuya propuesta curricular se desarrolla en espacios exteriores: el bosque, el campo, la playa o/y también en entornos urbanos. Es un modelo reglado que tiene su origen a mediados del siglo pasado en Dinamarca, donde en 1954 comenzó a funcionar la primera escuela infantil al aire libre ${ }^{\mathrm{I}}$. Desde allí se empezó a difundir este modelo por Escandinavia (Schaffert, 2004).

Hoy en día este modelo es habitual en muchos países europeos (Figura I). En Alemania, desde los años 90 hasta la actualidad han surgido unas 3000 escuelas que siguen este modelo ${ }^{2}$. Son escuelas de segundo ciclo de educación infantil, para niños y niñas de 3 a 6 años en Alemania y en Suiza incluso a veces hasta los 7 años. En su gran mayoría están homologadas como escuelas privadas concertadas, aunque según la Federación Nacional Alemana de los Jardines de Infancia en el Bosque (2019) existen también algunas experiencias públicas. Más frecuente aún es este modelo en el primer ciclo de educación infantil, comprendiendo las edades de i a 3 años, en las que se empieza a salir al medio natural en cuanto los niños y las niñas empiezan a caminar de forma autónoma. En Alemania estas experiencias durante la primera infancia son

En danés denominado Skovbørnebave, «jardín de infancia en el bosque».

En alemán denominado Waldkindergarten, «jardín de infancia en el bosque». 
BOSQUESCUELA.

UN MODELO DE ESCUELA SOSTENIBLE EN LA NATURALEZA PHILIP BRUCHNER Y ANTONIO ARAGÓN REBOLLO

conocidas como «grupos de juego en la naturaleza»³. Aunque se carece de una base oficial de datos para estos proyectos, se puede constatar que en muchos municipios alemanes, aunque sean pequeños, suele haber al menos uno o más proyectos de este tipo en el que niños y niñas pasan varias horas al día en la naturaleza.

En España, desde 2008, el autor de este artículo empezó a adaptar este modelo europeo a la legislación española y lo dio a conocer a través de conferencias, entrevistas de televisión, publicaciones en revistas y actividades formativas (Bruchner, 20I2; RTVE, 20I2). Gracias a la ayuda de una amplia red de colaboradores universitarios y contando con el apoyo institucional de la Fundación Félix Rodríguez de la Fuente se creó la primera escuela infantil al aire libre de España: el Centro Bosquescuela. Fue homologada por la Comunidad de Madrid y abrió sus puertas en 2015 en el municipio de Cerceda, a las puertas del Parque Nacional de la Sierra de Guadarrama (RTVE, 20I5; RTVE, 2OI6).

Hoy en día existen unos 40 proyectos de educación infantil al aire libre en España dentro de los cuales el Centro Bosquescuela Cerceda sigue siendo la única escuela homologada dentro del currículum español (In Natura, 2020). Debido a que la legislación española dificulta la homologación de este tipo de proyectos, desde el proyecto Bosquescuela se sigue haciendo mucho hincapié en el desarrollo del modelo y se han creado varias alianzas con entidades del sector privado y público para trabajar por la difusión y el reconocimiento oficial de este modelo pedagógico en varias comunidades autónomas: en 2018 la fundación Kutxa Ekogunea arrancó el proyecto «Basoeskola» con el fin de implementar el modelo en escuelas públicas en el País Vasco (Kutxa Ekogunea, 20I8). En el mismo año, Ecoembes creó el proyecto Naturaliza, en el cual está colaborando Bosquescuela y mediante el cual Ecoembes pretende ofrecer una educación ambiental trasversal en asignaturas troncales de Primaria: Ciencias de la Naturaleza, Ciencias Sociales, Matemáticas y Lengua Castellana (Ecoembes, 2020). En 2019, el Centro Universitario La Salle, adscrito a la Universidad Autónoma de Madrid, homologó un amplio programa de formación en Metodología Bosquescuela como Programa Superior Universitario que fue también acreditado por la Consejería de Educación e Investigación de la Comunidad de Madrid. Desde entonces son muchos los profesionales que se han formado en esta metodología y que promueven que en sus centros ya se estén naturalizando los espacios y aumentan los proyectos en contacto con la naturaleza (Muela et al., 2019). Pero para su implementación sigue siendo necesario cambiar o amoldar la legislación como ya es habitual en muchos otros países de Europa y del mundo (Bruchner, 20I7).

\section{Fundamentos didácticos y estilo de formación}

El modelo Bosquescuela tiene una serie de fundamentos y aspectos que permiten justificar la naturaleza como aula y que hacen de él un modelo relevante para la educación en la etapa infantil:

I. La naturaleza juega un papel extraordinariamente importante. Como aula, la naturaleza cuenta con una característica muy especial: está viva. Bruchner (2017) destaca que en la naturaleza todo está «en un continuo proceso de cam-

3 En alemán «Waldspielgruppen». 
bio debido a la influencia de varios factores como el tiempo, las estaciones del año, la vida y el crecimiento, la descomposición, el nacimiento y la muerte y la radiación solar» (p. 44). De esta forma, los niños y las niñas y los maestros y las maestras acompañantes se encuentran con contextos cambiantes cada día. Además, se enfrentan a diario a un entorno que está caracterizado por una «inmensa variedad de terrenos y espacios, de plantas, animales, piedras y suelos con una gran amplitud de texturas, colores, superficies, formas y pesos» (Bruchner, 20I7, p. 44). Se ha observado en el día a día del funcionamiento del Centro Bosquescuela Cerceda que, debido a la inmensa variedad de recursos del entorno natural y su carácter vivencial y desestructurado, cada uno de los alumnos y alumnas tiene la oportunidad de encontrar los estímulos individuales necesarios que conectan con cada uno de sus intereses, favoreciendo así que esa experiencia vivencial acompañe su desarrollo de la forma más adecuada (Figura 2).

2. Se producen vivencias imprevistas y espontáneas fruto de las interacciones entre la infancia y su entorno. El carácter vivencial de estas interacciones mantiene viva la atención del alumnado generando actitudes de curiosidad y conectando así con las motivaciones e intereses intrínsecos de los niños y las niñas. Esto supone que los aprendizajes individuales de los niños y las niñas sean del todo significativos y queden grabados y permaneciendo en el tiempo de forma duradera. Además, Miklitz (2000) señala que el mismo entorno de la naturaleza ofrece la posibilidad de vivir procesos que se llevan a cabo de forma autónoma, es decir, los materiales y los espacios se estructuran a sí mismos sin la influencia directa del ser humano y por eso está lleno de sorpresas, otro aspecto fundamental que hace tan ricos los procesos de aprendizaje.

3. Varios autores consideran que la educación al aire libre facilita la conexión de los niños y las niñas consigo mismos; el desarrollo de la sensación de competencia personal, y la creación de una imagen saludable de sí mismos y de su relación con el mundo que les rodea (American Institutes of Research, 2005; O'Brien, 2009). La riqueza y la calidad de los estímulos naturales también tiene su efecto positivo sobre la integración sensorial del niño, un concepto que fue estudiado por la neurobióloga americana Jean Ayres. Ayres (2006) señaló que una integración sensorial correcta es condición indispensable para que los niños y las niñas sean capaces de desarrollar competencias importantes como la lectoescritura, el desarrollo del sentido lógico-matemático o la concentración y la atención sostenida (Figura 3).

4. Se dan oportunidades en las que los niños y las niñas salen de su zona de confort. En el bosque cuando cruzan un río o cuando trepan a un árbol de forma natural y sin sentirse forzados, los niños y niñas se enfrentan a situaciones de conflicto cognitivo que, por su propio carácter, requieren de creatividad para desarrollar estrategias y encontrar las soluciones oportunas (Figura 4). En muchas de estas ocasiones además existe un cierto riesgo que, para superar el reto con éxito, requiere de una implicación de responsabilidad que, en otro contexto escolar, no se suele encontrar (Figura 5). Por lo tanto, Ball (2002), Bjorklund y Pellegrini (2002) son de la opinión de que el riesgo se entiende como un concepto inherente al aprendizaje en la naturaleza ya que los niños lo necesitan para su desarrollo emocional, social y cognitivo. Sandseter (2008) 

PHILIP BRUCHNER Y ANTONIO ARAGÓN REBOLLO

ha detectado y clasificado estas situaciones de riesgo y que sirven a los niños y las niñas como situaciones de aprendizaje. En el modelo Bosquescuela estas situaciones están vividas con normalidad a diario.

5. Se da mucha importancia a la motivación genuina, entendida según Largo (1999) como el deseo de aprender que nace genuinamente con base en la curiosidad. La curiosidad, innata en el ser humano, se manifiesta a través de los intereses del niño, sus preguntas, sus acciones, sus impulsos. En ese sentido se parte de que el aprendizaje no está limitado a acciones como aprender de memoria o repetir un contenido, sino que se alcanza con mayor probabilidad de éxito cuando tiene lugar a través de la interacción con y dentro del medio natural, a través del juego, de la comunicación, de la exploración y del movimiento. El aprendizaje a partir de experiencias reales se considera según varios autores como principio básico para trabajar contenidos, desarrollar competencias y adquirir habilidades (Tillman, Button, Coen y Gilliland, 20I9). La naturaleza además es el entorno perfecto para vivir experiencias directas y reales tras las cuales, el niño o la niña pueden explorar, investigar, moverse, estudiar o desarrollar cualquier actividad que les surja desde su motivación genuina (Coates y Pimlott-Wilson, 20I9; O’Brian, 2009). Con el objeto de identificar los aspectos concretos de esta motivación intrínseca, es decir, para identificar cuáles son las cosas que despiertan la motivación en el grupo y en qué punto de su desarrollo evolutivo se encuentran, Bosquescuela ha desarrollado un sistema de observación y documentación cualitativa. Con ello se pretende identificar, mantener y mejorar la calidad educativa y también desarrollar propuestas pedagógicas que permitan a los niños y a las niñas mantener viva su curiosidad en Bosquescuela. Este sistema de observación cualitativa se encuentra descrito en la Propuesta Pedagógica del Centro Bosquescuela Cerceda (Bosquescuela Cerceda, 2015a).

6. Completando estos aspectos de fundamentación pedagógica es importante destacar que, en la educación infantil al aire libre, tal y como se lleva a cabo en los países nórdicos, mejora, según Bentsen, Mygind y Randup (2009) la interacción social. Hay que tener también en cuenta la alta heterogeneidad y la diversidad intrapersonal e interpersonal respecto al desarrollo individual y las necesidades de cada niño y niña. Por este motivo, el alumnado no está separado por edades, sino que se mezclan, y los niños y las niñas de 3 a 5 años forman una clase unitaria acudiendo todos al mismo grupo. De esta manera heterogénea, se aprovecha la riqueza y diversidad en todas las situaciones de aprendizaje que se dan en el día a día como también opinan Kucharz y Wagner (2007).

Se puede concluir que Bosquescuela es un modelo de escuela sostenible no solo por darse en un entorno vivo y diverso, sino porque el exterior como entorno de aprendizaje contribuye de manera vivencial y emocional a la adquisición de valores de respeto hacia uno mismo, al otro y al entorno y a interiorizar las interrelaciones de los ciclos naturales. Ambas cuestiones son fundamentales en la educación para la sostenibilidad (Aragón, 2109 y Rodríguez et al., 2020).

\section{Realidad concreta del Centro Bosquescuela Cerceda}

El Centro Bosquescuela Cerceda cuenta con una unidad infantil con 25 plazas para niños y niñas de 3 a 5 años. 
BOSQUESCUELA.

UN MODELO DE ESCUELA SOSTENIBLE EN LA NATURALEZA

PHILIP BRUCHNER Y ANTONIO ARAGÓN REBOLLO

\section{I. Objetivos}

En una escuela infantil al aire libre-siendo homologada- se parte de los objetivos que están formulados en el currículum oficial de educación infantil. En Bosquescuela Cerceda en concreto, se respeta y se cumple con todos los principios y fines educativos que marca la Ley Orgánica 8/2013, de 9 de diciembre, para la mejora de la calidad educativa (LOMCE 8/20I3) y el Decreto I7/2008, del 6 de marzo, del Consejo de Gobierno (Decreto $17 / 2008$, de 6 de marzo), por el que se desarrollan para la Comunidad de Madrid las enseñanzas de la Educación Infantil, especialmente en atención a los objetivos que se definen para la Educación Infantil y la formación del profesorado.

\subsection{Recursos - el aula}

El Centro está ubicado en una parcela municipal que se encuentra dentro del monte público de Cerceda, la Dehesa Boyal (Figura 6). La Dehesa, que cuenta con una extensión de unas 70 ha, ofrece encinares, fresnedas y bosque de ribera. Todos los sitios de interés están situados a distancias razonables entre sí para realizar los paseos diarios con los niños y niñas. Por otra parte, el lugar está muy bien comunicado en transporte público a través de varias líneas de autobuses que permiten llevar a cabo excursiones semanales a otros espacios naturales o a entornos urbanos como centros culturales, teatros, museos, etc.

\subsection{Recursos-el personal}

La ratio numérica de alumnado/profesorado es muy baja: cuando el número de niños y niñas por unidad sea igual o inferior a I6, contarán con 2 docentes. En unidades con más de 16 niños y niñas los acompañantes serán 3 docentes.

Se ha incorporado en el currículo escolar del centro el aprendizaje de la lengua inglesa mediante la inmersión lingüística. Esta es impartida por un profesional que posee el título de Grado en Educación Infantil o el título de Maestro con la especialidad de Educación Infantil con la acreditación en inglés. Además, el centro cuenta con I auxiliar nativo inglés que se comunica con los niños y las niñas durante toda la jornada en su lengua materna.

Los maestros y las maestras son las figuras de referencia: son quienes realizan propuestas y llevan la responsabilidad de la calidad de las interacciones sociales entre todos los miembros de la comunidad educativa. Los maestros y las maestras se relacionan con los niños y las niñas, respetando su integridad personal y tratándoles con la igualdad y dignidad que Juul (2004) considera esencial. Sus funciones incluyen definir y asegurar límites físicos y emocionales que generen un contexto seguro para asegurar que las necesidades y derechos de la infancia y de todas las personas que participan en el proceso educativo del centro están cubiertas. Estos límites se llevan a cabo de forma respetuosa y sin violar la integridad de la infancia. Ante los conflictos extremos, los maestros y las maestras actúan para proteger y cuidar emocionalmente a las personas implicadas, validando los sentimientos y emociones y ayudando a los niños y las niñas a utilizar el lenguaje para describir situaciones, emociones y buscar 
BOSQUESCUELA.

UN MODELO DE ESCUELA SOSTENIBLE EN LA NATURALEZA PHILIP BRUCHNER Y ANTONIO ARAGÓN REBOLLO

soluciones a conflictos, sin tomar partido ni realizar juicios y nunca para castigar, siguiendo las bases de la Comunicación No Violenta según Rosenberg (2006).

\subsection{Recursos - el refugio}

En las escuelas infantiles al aire libre se carece conscientemente de instalaciones tradicionales. Si estas existen, suelen ser de reducidas dimensiones.

En el Centro Bosquescuela Cerceda se cuenta con una cabaña de madera que sirve como punto de encuentro por las mañanas y de recogida por las tardes y, a menudo, para descansar al mediodía (Figura 6). Bajo condiciones meteorológicas extremas (tormenta, ventisca, granizo o si hace mucho calor) niños, niñas, maestros y maestras pueden buscar refugio en esta cabaña hasta que el tiempo mejore. La cabaña dispone de una superficie útil de $50 \mathrm{~m}^{2}$. El equipamiento de la cabaña es similar al del resto de las cabañas de las escuelas infantiles al aire libre al uso en Alemania. Para días de mucho calor, la edificación dispone de un aislamiento extra en el tejado y un toldo en el porche. Para las estaciones frías dispone de una estufa de leña que calienta la cabaña rápidamente.

En la cabaña existen diferentes materiales didácticos a disposición del grupo como pinturas, libros, papeles, tijeras, plastilina, telas, marionetas, herramientas de madera, bloques de construcciones, etc., que se usan para las fases de horas no lectivas como el descanso o la llegada/recogida. También hay mesas y bancos plegables que se utilizan indistintamente tanto para comer como para llevar a cabo diferentes propuestas pedagógicas como los talleres de arte o para que los niños y las niñas puedan utilizar los diferentes materiales que hay disponibles en la cabaña (Figura 7). En la parte superior al fondo de la cabaña hay un altillo, con barrera de protección y accesible por una escalera, en el que aquellos que lo necesiten pueden dormir la siesta tras la hora de comer.

El impacto medioambiental de la cabaña es ínfimo. Se trata de una cabaña bioclimática con huella cero de $\mathrm{CO}_{2}$ que está construida de madera de abeto Douglas y de alerce, dos maderas que resisten de forma natural a los insectos y hongos sin la necesidad de recibir tratamiento con pesticidas. Carece de electricidad. No hace falta luz ya que la escuela está abierta solo durante el día y la orientación espacial de la cabaña está diseñada de tal manera que el sol ilumine y caliente de forma modesta y óptima la instalación durante todo el año. Para días en invierno se usa una estufa de leña como apoyo para calentarla puntualmente. Además, dispone de dos wc orgánicos de compostaje, uno para niños y niñas y otro para adultos. De esta forma ni gasta ni contamina el agua.

\subsection{Propuesta curricular}

El currículo se lleva a cabo de forma flexible y globalizada y se propone a través de ciertas rutinas (ver más adelante abajo) que ayudan a los niños y las niñas a orientarse temporalmente, y les aporta un marco de seguridad y la adquisición de pequeños hábitos, según se detalla en el Proyecto Educativo de Centro Bosquescuela Cerceda (Bosquescuela Cerceda, 20r5b). Los maestros y las maestras parten de estos elementos 
rutinarios para llevar a cabo propuestas pedagógicas que dan margen para desarrollar los objetivos y contenidos definidos para la etapa de Educación Infantil en el Anexo I del Decreto 17/2008, de 6 de marzo, de la Comunidad de Madrid (Decreto I7/2008, de 6 de marzo).

El objetivo es la adquisición de las competencias clave. Para conseguirla se aborda el currículum escolar de una manera comunicativa, activa, participativa, adaptada a los distintos ritmos de aprendizaje, favoreciendo que sea el alumnado quien aprenda por sí mismo y de manera cooperativa y colaborativa, especialmente dirigida a contribuir a la adquisición de estas competencias clave que están recogidas en la Orden $\mathrm{ECD} / 65 / 2015$, de $2 \mathrm{I}$ de enero, por la que se describen las relaciones entre las competencias, los contenidos y los criterios de evaluación de la educación primaria, la educación secundaria obligatoria y el bachillerato:

SIEE. Sentido e iniciativa y espíritu emprendedor: Desarrollando la capacidad de liderazgo y de trabajo en equipo, así como el sentido crítico y de la responsabilidad tratando desde una perspectiva más holística el concepto de cambio climático.

CEC. Conciencia y expresiones culturales: Promocionando la participación en la vida y la actividad cultural en la sociedad en la que se vive, favoreciendo la convivencia social teniendo en cuenta la importancia del medio natural con el que nos interrelacionamos.

AA. Aprender a aprender: Facilitando a través del juego cooperativo un medio en el cual puedan conectar los conocimientos previos para ir desarrollando otros nuevos conocimientos a través de la interacción con todo el grupo con las diferentes actividades planteadas a lo largo del juego, a lo que también dan importancia los autores Coates y Pimlott-Wilson (2019).

CL. Competencia en comunicación lingüística: Fomentando las destrezas y estrategias comunicativas para la lectura, la escritura, el habla, la escucha y la conversación a través de las diferentes modalidades que ofrece el juego cooperativo con temática de respeto y cuidado por el medio ambiente (Lieberman y Hoody, I998; O’Brien, 2009; Streelasky, 2019).

Csc. Competencias sociales y cívicas: Activando mecanismos para comprender y entender cómo las experiencias colectivas modifican el medio ambiente y la importancia de repensar sus consecuencias. Fomentado la capacidad de la cooperación y los compromisos para afrontar conflictos y proponer soluciones para enfrentarlos.

смст. Competencia matemática y competencias básicas en ciencias y tecnología: Proporcionando un acercamiento a los métodos propios de la actividad científica: propuesta de preguntas, búsqueda de soluciones, indagación de caminos posibles y para la resolución de problemas relacionados con el cambio climático (García González y Schenetti, 2019).

\subsection{Rutinas}

Al inicio de curso, en el Centro Bosquescuela Cerceda se establece una propuesta semanal estable para todo el curso escolar, en la que cada día tiene su estructura diaria definida. En Cerceda se distinguen 3 tipos de días: lunes, martes y miércoles se realizan paseos por la Dehesa Boyal. Los jueves se realiza una excursión fuera del contexto natural para favorecer eperiencias de la vida urbana. Como ejemplo se visita 
BOSQUESCUELA.

UN MODELO DE ESCUELA SOSTENIBLE EN LA NATURALEZA PHILIP BRUCHNER Y ANTONIO ARAGÓN REBOLLO

el pueblo de Cerceda, al que se llega andando desde el centro o se coge el autobús para conocer otras localidades de la zona o de la provincia (Figura 8). Los viernes es el «día de taller», donde el grupo se queda en la proximidad de la cabaña para llevar a cabo talleres de diferentes temáticas como, por ejemplo, música, arte o filosofía infantil (Figuras 7, 9 y io).

Cada uno de estos tres tipos de días tiene sus propios tiempos definidos dentro de los cuales se distinguen un total de trece elementos rutinarios distintos. Cada uno de estos elementos está caracterizado por distintas cualidades que permiten a los niños y las niñas la adquisición de los contenidos propuestos en el currículum.

Se identifican los siguientes 6 elementos rutinarios:

I. Fase de llegada/recogida

Por la mañana las familias llegan con los niños y las niñas a la cabaña, que es el punto de encuentro. Por la tarde les recogen en el mismo lugar. Durante estas fases no lectivas, los niños y las niñas pueden hacer uso de todos los materiales que les ofrece la cabaña y sus alrededores. Hay mesas en las que pueden trabajar dentro o fuera de la cabaña. Igualmente pueden jugar en los alrededores directos de la cabaña aprovechando los recursos y espacios que ofrece el medio natural. Tanto la fase de acogida como la de despedida dura aproximadamente media hora (Figura II).

2. Actividad de bienvenida

La actividad de bienvenida se lleva a cabo todos los días en el terreno situado alrededor de la cabaña. Se da la bienvenida al grupo y a los maestros y las maestras. Se cantan canciones, se hacen juegos de movimiento o se baila. Esta actividad dura aproximadamente un cuarto de hora.

3. Clases

Las clases se llevan a cabo al aire libre, en el terreno aledaño a la cabaña o durante el paseo hacia el lugar del día. Se proponen unidades didácticas que acogen los contenidos relacionados con las tres áreas educativas del currículo del $2{ }^{\circ}$ ciclo de Educación Infantil de manera significativa y constructivista (Figura I2). Unas características principales de trabajar las clases en plena naturaleza son las sorpresas que depara cada día: una cigüeña, un águila calzada o simplemente una lombriz que uno de los niños o las niñas encuentra en el suelo. Por ello, trabajar en el medio natural requiere una alta flexibilidad y creatividad por parte del profesorado (Figuras I3 y I4). La duración de una clase puede variar entre is y 60 minutos.

4. Paseo al lugar del día

El «lugar del día» es un lugar situado a una distancia de entre 200 metros y un kilómetro de la cabaña (Figura I5). Los maestros y las maestras eligen un camino con retos que alcanzar. A lo largo de este paseo se suelen hacer múltiples descubrimientos, se inician pequeñas investigaciones, los niños y las niñas conectan de forma individual con sus inquietudes e intereses, es el llamado «camino del aprendizaje» (Figura I6). Todos los aprendizajes en este momento son significativos. El paseo al lugar del día se prolonga aproximadamente durante media hora. El equipo tiene predefinidos varios «lugares del día» que se establecen y definen a lo largo del año escolar y se van visitando en función de la distancia a la cabaña y la meteorología. 
5. Picnic

Ya en el lugar del día, el grupo se sienta en círculo sobre aislantes en el suelo. Se inicia un pequeño ritual que introduce el momento del almuerzo, se propone cantar una canción y después niños, niñas y adultos acompañantes sacan los almuerzos que han llevado en sus mochilas desde casa. Es un momento de calma y placer compartido en el que cada niño y niña se toma su tiempo y conecta con sus necesidades básicas de bienestar (Figura I7). El momento del picnic dura aproximadamente media hora con la flexibilidad de que haya niños o niñas que necesiten de más tiempo. A medida que cada uno va terminando su almuerzo, guarda sus cosas con total autonomía en la mochila y se dispone a comenzar el momento del juego espontáneo y libre.

6. Juego espontáneo y libre

Durante el juego espontáneo y libre los niños y niñas eligen a qué, con quién y dónde quieren jugar (Figura I8). El juego espontáneo y libre es el pilar del proyecto Bosquescuela y se considera como la propuesta pedagógica que mejor se adapta al desarrollo de la infancia, a las características individuales de cada uno de los niños y las niñas, a sus necesidades, a sus intereses y al estilo cognitivo de cada uno de ellos. Cada niño y niña puede aprender de forma distinta según sus propias motivaciones y fortalezas. En el juego espontáneo y libre, entendido como propuesta pedagógica, se favorece especialmente la atención a la diversidad y la atención individualizada ya que cada uno de los niños y las niñas es protagonista de su propio aprendizaje y desarrollo; así, a través de sus juegos, crean su propio currículo apoyado y acompañado por los maestros (Coates, 20I9; Kiewra y Veselack, 20I6). La fase del juego libre suele tener una duración de 2 horas y termina con una narración oral que los maestros suelen llevar a cabo a diario (Figura i9).

7. Regreso a la cabaña, comedor y descanso

Después del juego libre, el grupo regresa a la cabaña donde algunos niños y niñas se encuentran con sus familias y otros comienzan a prepararse para el momento de la comida y el descanso que se realiza dentro de la cabaña. A las I6:30 horas la escuela cierra hasta el día siguiente.

Respecto a la realidad concreta de un centro Bosquescuela se puede concluir que el desarrollo del currículo escolar se realiza de manera trasversal a través de rutinas que consideran todo el entorno como escenario de aprendizaje. Con ello se busca que las competencias clave se adquieran de una manera comunicativa, activa, participativa, adaptada a los distintos ritmos de aprendizaje, y favoreciendo que sea el alumnado quien aprenda por sí mismo y de manera cooperativa y colaborativa.

\section{Educación de calidad para un Desarrollo Humano Sostenible}

El desarrollo sostenible tiene en la actualidad un nuevo horizonte en forma de Agenda 2030 que se enmarca en los 17 Objetivos de Desarrollo Sostenible (oDs) adoptados por la ONU (ONU, 20I5a) para erradicar el cambio climático y asegurar la salud y prosperidad para todos. Este marco se resume en la idea de desarrollo sostenible que se definió en el informe Brundland, «Nuestro futuro Común», en r987 y que el Libro 

PHILIP BRUCHNER Y ANTONIO ARAGÓN REBOLLO

Blanco de la Educación ambiental recoge como aquel desarrollo que asume la existencia de límites físicos que sugieren que es imposible un crecimiento sin fin; enfatiza la necesidad de mejorar la calidad de vida, frente al bienestar basado en los bienes materiales, y se inspira en la solidaridad entre las diferentes generaciones y entre los individuos de cada generación (Ministerio de Medio Ambiente, 1999).

El desarrollo del concepto de la sostenibilidad en Bosquescuela se lleva a cabo desde el principio de que la percepción es previa al pensar, de tal manera que vamos a pensar el mundo en función de cómo lo percibimos. Esto es crucial en la primera infancia ya que es la etapa en la que empezamos a descubrirnos a nosotros mismos, al otro y al entorno en el que vivimos y en la que más conexiones neuronales se producen. Por ello, se trabaja en la percepción de uno para conocerse más fácilmente y así ser más consciente de cuáles son los recursos materiales, emocionales, etc., que el niño o la niña requiere para satisfacer sus necesidades. Con ello, se evita, en primer lugar, derrochar esfuerzos y recursos que no contribuyen a su bienestar y, en segundo lugar, se trabaja la toma de decisiones que influye directamente en el plano económico de la sostenibilidad, que no es otro que el de la «administración de la casa». Economía significa literalmente «dirección y administración de la casa» (que viene del griego oikonomía, que incluye oikos-casa), y los niños y las niñas entienden que la primera casa es el propio cuerpo. Así, es más fácil satisfacer las necesidades reales y con ello ser más fácil, más pleno y feliz.

Pero esta felicidad no depende de uno mismo, sino que vivimos con otras personas, por lo que es fundamental trabajar nuestras emociones sociales, base de la empatía que a su vez nos lleva a tolerar al otro como alguien diferente pero igual en dignidad. Este hecho tiene un gran protagonismo en el modelo Bosquescuela, ya que se trabaja siempre la resolución de conflictos mediante una comunicación emocional y no violenta, como antes ya se ha mencionado, basándose en dos referentes: Juul (2004) y Rosenberg (2006), quienes destacan estos aspectos importantes para la creación de las relaciones entre las personas. Con ello se está contribuyendo a generar en los niños y las niñas un cambio en la percepción del otro como de alguien con quien comparto un tiempo y un espacio a ser alguien con quien convivo de una manera respetuosa, rica, en la que se establecen dinámicas de cuidado y responsabilidad para con él o ella; se contribuye a crear sentimiento de comunidad, y se establecen fuertes vínculos que generan tejido social (Bentsen, Mygind y Randup, 2009; Chawla et al., 2014; Coates y Pimlott-Wilson, 2019; O’Brien, 2009; Wistoft y Dyg, 2018).

Y, por último, conocer el medio que nos rodea de forma vivencial no solo para entender sus procesos, sino que a través de la experimentación en él y con él permite crear un vínculo fundamental para habitar el mundo, generando actitudes de empatía y respeto hacia el medio ambiente, respetándolo como una parte de nuestro desarrollo personal, como el lugar donde voy a vivir toda la vida, un aspecto fundamental que también han investigado Browning y Rigolon (2019). En el modelo Bosquescuela los oDs están presentes en su funcionamiento diario. Por ejemplo, el tipo de instalaciones con emisiones cero hace que los niños y las niñas experimenten en primera persona el ODS I3, que pretende educar sobre el cambio climático.

Por otro lado, las dinámicas de juego que se establecen en la naturaleza no tienen condicionantes de género y la promoción de la comunicación no violenta y respetuosa promueve la igualdad de género que marca el oDs 5. De hecho, la naturaleza como tal tiene de manera intrínseca criterios que se buscan en el diseño de patios inclusivos, 
BOSQUESCUELA.

UN MODELO DE ESCUELA SOSTENIBLE EN LA NATURALEZA PHILIP BRUCHNER Y ANTONIO ARAGÓN REBOLLO

tales como diversidad de ambientes, diversidad de propuestas deportivas, diversidad de niveles y promoción de la autonomía (Cols y Fernández, 2019), el bienestar personal (Corraliza y Collado, 2016) y la equidad (Lagar, 20I5).

Durante las excursiones surgen conflictos y retos que requieren la cooperación para ser solucionados, como ayudar a cruzar un río, transportar troncos grandes, y se repiten dinámicas de cuidado y acompañamiento de los mayores hacia los más pequeños. Estos y otros ejemplos similares permiten a nuestro juicio vivenciar la necesidad de generar alianzas para superar los retos que ofrece el entorno natural a diario, que facilita el entendimiento del ODS I7 sobre la necesidad de generar alianzas para lograr los ODs. Mucho más directa aún es la vivencia del ods I5, que pretende promover la gestión sostenible de todos los tipos de bosques, la conservación de los ecosistemas terrestres al sentir estos como parte de su vida como también varios autores lo señalan (Chen y Monroe, 20I2; Freire, 20II; Hartig et al., 20I4; Nisbet, Zelenski y Murphy, 20II; Ward, Aspinall y Montarzino, 2007; Wells y Lekies, 2006).

Pero, si hay un oDs que tiene un protagonismo especial en el modelo Bosquescuela, este es el oDs 4 sobre educación de calidad. Concretamente, la contribución que este hace a la meta 4.7, que plantea la necesidad de que «de aquí a 2030, garantizar que todos los alumnos adquieran los conocimientos teóricos y prácticos necesarios para promover el desarrollo sostenible, entre otras cosas mediante la educación para el desarrollo sostenible y la adopción de estilos de vida sostenibles, los derechos humanos, la igualdad de género, la promoción de una cultura de paz y no violencia, la ciudadanía mundial y la valoración de la diversidad cultural y de la contribución de la cultura al desarrollo sostenible» (ONU, 20isb).

Por consiguiente, partiendo de la experimentación en el medio natural que sigue la metodología que se lleva a cabo en Bosquescuela se puede contribuir a introducir en el alumnado la cultura de la sostenibilidad en la que deben confluir los planos económicos, social y ambiental. Esto es así gracias a que en el día a día se genera en ellos una visión de sí mismo, del otro y del mundo consciente y respetuosa. Con ello se trabaja la sociabilidad compartida como individuos que forman parte de un grupo que se siente comunidad, cuyos vínculos se basan en el cuidado de uno mismo, del otro y del entorno (Aragón, 20I4). De esta manera se establece desde la infancia el desarrollo de una vida personal más saludable (se trabajan problemas tales como el acoso escolar, desigualdad de género, el déficit de naturaleza, la obesidad, entre otros), plena, consciente y feliz, que deriva en una convivencia más respetuosa, tolerante e inclusiva y que genera comportamientos de respeto y cuidado mayor del medio ambiente.

Así, en el modelo Bosquescuela no solo están presentes de manera implícita los oDs, sino que también se trabajan algunos de los retos educativos que, según Heras, Marcen y Benayas (2019), derivan de la crisis socioambiental y climática: articular lo local y lo global, incluir la perspectiva social en la problemática ambiental mostrando que el ser humano forma parte de la naturaleza y los problemas ambientales son problemas socioambientales, integrar lo individual y lo colectivo y aproximarse a la complejidad de las relaciones que se dan en la naturaleza.

Pese a que no es un modelo nuevo, Bosquescuela es un modelo vigente que puede contribuir de manera innovadora a cumplir los objetivos de la agenda 2030 desde la etapa infantil.

Por tanto, se puede considerar como un modelo ejemplar de buenas prácticas para educar en la sostenibilidad. 
BOSQUESCUELA.

UN MODELO DE ESCUELA SOSTENIBLE EN LA NATURALEZA

PHILIP BRUCHNER Y ANTONIO ARAGÓN REBOLLO

\section{Bibliografía}

American Institutes of Research. (2005). Effects of Outdoor Education Programs for Children in California. Sacramento: California Department of Education.

Aragón Rebollo, T. (2014). Reconfiguración del paisaje desde lo común. Una perspectiva ético-estética. Enrahonar. Quaderns de Filosofia, 53, 43-6I.

Aragón Rebollo, T. (2019). Aportes prácticos desde la filosofía, la psicología y la neurociencia para generar cultura de la sostenibilidad desde la Educación Ambiental en un contexto de ods, emergencia climática y ciudadanía global. En A. Barrón Ruiz y J. M. MuÑoz RoDRÍGUEZ (coords.), Crear y hacer educación ambiental. XXI Seminario de Investigación en educación ambiental (pp. 40-62). Ed. Organismo Autónomo Parques Nacionales. Madrid: Ministerio para la Transición Ecológica y Cambio Demográfico.

Ayres, A. J. (2006). La integración sensorial y el niño. Sevilla: Editorial Mad S.L.

Ball, D. J. (2002). Playgrounds - Risks, benefits and choices (vol. 426). Londres: Health and Safety Executive (HSE) contract research report, Middlesex University.

Bentsen, P.; Mygind, E. y Randrup, T. (2009). Towards an understanding of udeskole: Education outside the classroom in a Danish context. Education, 3(13), 37, 29-44. IO.IO80/03004270802291780.

Bjorklund, D. F. y Pellegrini, A. D. (2002). The origins of human nature: Evolutionary developmental psychology. Washington, D.C.: American Psychological Association.

Bosquescuela Cerceda. (20I5a). Propuesta Pedagógica del Centro Bosquescuela Cerceda $(P P C)$. Cerceda: Bosquescuela Cerceda.

Bosquescuela Cerceda. (20i5b). Proyecto Educativo del Centro Bosquescuela Cerceda (PeC). Cerceda: Bosquescuela Cerceda.

Browning, M. y Rigolon, A. (2019). Could nature help children rise out of poverty? Green space and future earnings from a cohort in ten U.S. Cities. Environmental Research, 176, I08449. https://doi.org/IO.I0I6/j.envres.2019.04.016

Bruchner, P. (2012). Escuelas infantiles al aire libre. Cuadernos de Pedagogía, 420, 26-29.

Bruchner, P. (20I7). Bosquescuela: Guía para la educación infantil al aire libre. Cullera (Valencia): Ediciones Rodeno.

Chawla, L.; Keena, K.; Pevec, I. y Stanley, E. (20I4). Green schoolyards as havens from stress and resources for resilience in childhood and adolescence. Health Place, 28, I-I3. doi: I0.I0I6/j.healthplace.2014.03.00I

Chen, J. H. y Monroe, M. C. (2012). Connection to Nature: Children's Affective Attitude Toward Nature. Environment and Behavior, 44(I), 3I-49. https://doi. org/Io.1177/0013916510385082

Coates, J. K.y Pimlotт-Wilson, H. (2019). Learning while playing: Children's Forest School experiences in the UK. British Educational Research Journal, 45(I), 2I-40. doi: http://dx.doi. org/Io.I0o2/berj.349I

Cols Clotet, C. y Fernàndez Quiles, J. (Pitu). (2019). Patios inclusivos. Tarbiya, Revista de Investigación e Innovación Educativa, (47), I07-II5. https://doi.org/IO.I5366/tarbiya2019.47.008

Comisión Mundial del Medio Ambiente y sel Desarrollo. (i988). Nuestro Futuro Común. Madrid: Alianza Editorial.

Corraliza, J. A. y Collado, S. (2016). Conciencia ecológica y bienestar en la infancia. Efectos de la relación con la naturaleza. Madrid: Editorial CSS.

Consejería de Educación. (2008, i2 de marzo). DECRETO 17/2008, de 6 de marzo, por el que se desarrollan para la Comunidad de Madrid las enseñanzas de la Educación Infantil. Boletín Oficial de la Comunidad de Madrid, n. ${ }^{\circ}$ 6r. Recuperado el 23 de noviembre de 2020, de http://www.madrid.org/wleg_pub/secure/normativas/contenidoNormativa.jsf?opcion=VerHtmlynmnorma $=4922$ cdestado $=$ P\#no-back-button . 
BOSQUESCUELA.

UN MODELO DE ESCUELA SOSTENIBLE EN LA NATURALEZA

PHILIP BRUCHNER Y ANTONIO ARAGÓN REBOLLO

Dickie, I.; Ozdermiroglu, E. y Phang, Z. (20io). Assessing the benefits of learning outside the classroom in natural environments, Final Report for Natural England. Recuperado el 23 de noviembre de 2020, de https://ecosystemsknowledge.net/sites/default/files/wp-content/ uploads/2013/03/Learning-in-Natural-Environments-report-2802II.pdf.

Ecoembes. (2020). Naturaliza. Nuestro proyecto. Madrid: Ecoembes. Recuperado el 23 de noviembre de 2020, de https://www.naturalizaeducacion.org/nuestro-proyecto/.

Federación Nacional Alemana de los Jardines de Infancia en el Bosque (en alemán «Bundesverband der Natur- und Waldkindergärten in Deutschland e.V.»). (20I9). Deutschland. Recuperado el 23 de noviembre de 2020, de https://bvnw.de/deutschland/.

Freire, H. (2OII). Educar en verde. Ideas para acercar a niños y niñas a la naturaleza. Barcelona: GRAÓ de IRIF.

García González, E. y Schenetti, M. (2019). Las escuelas al aire libre como contexto para el aprendizaje de las ciencias en infantil. El caso de la Scuola nel Bosco Villa Ghigi. Revista Eureka sobre Enseñanza y Divulgación de las Ciencias, I6(2), 2204.

Hartig, T.; Mitchell, R.; De Vries, S. y Frumkin, H. (20I4). Nature and Health. The influence of nature on social, psychological and physical well-being. Annual Review of Public Health, 35:1, 207-228.

Heras, F.; Marcén, C. y Benayas, J. (20I9). Educación, ambiente y sostenibilidad: reflexiones y perspectivas. En J. BENAYAS y C. MARCÉN (coords.). Hacia una educación para la sostenibilidad. 20 años después del Libro Blanco para la educación ambiental (pp. 385-399). Madrid: Red Española para el Desarrollo Sostenible y Ministerio para la Transición Ecológica-Gobierno de España. Recuperado el 23 de noviembre de 2020, de https://www.miteco. gob.es/es/ceneam/recursos/materiales/hacia-educacion-sostenibilidad_tcm30-496569.pdf.

In Natura. (2020). Directorio de Escuelas en la Naturaleza. Madrid: In Natura. Recuperado el 23 de noviembre de 2020, de https://escuelainnatura.com/directorio-escuelas-en-la-naturaleza-espana/.

JuUl, J. (2004). Su bijo una persona competente. Barcelona: Herder.

KAPLAN, S. (1995). The restorative benefits of nature: Toward and integrative framework. Journal of Environmental Psychology, I5, I69-182. https://doi.org/10.1016/0272-4944(95)900oI-2

Kiewra, C. y Veselack, E. (20I6). Playing with nature: Supporting preschoolers' creativity in natural outdoor classrooms. The International Journal of Early Childhood Environmental Education, 4(I), 7I-96.

Kucharz, D. y Wagner, M. (2007). Jahrgangsübergreifendes Lernen. Eine empirische Studie zu Lernen, Leistung und Interaktion von Kindern in der Schuleingangsphase. Baltmannsweiler: Schneider Hohengehren.

Kutxa Ekogunea. (2018). Proyecto Bosquescuela. Hernani: Fundación Kutxa Ekogunea. Recuperado el 23 de noviembre de 2020, de https://www.ekogunea.eus/es/ekogunea/hezkuntza-programa/basoeskola-proiektua/3-7-47/.

Lagar, G. (2015). Patios y Parques Dinámicos. España: Trabe.

LARgo, R. H. (1999). Kinderjabre: Die Individualität des Kindes als erzieherische Herausforderung. München: Piper.

España. (Io de diciembre de 20I3). Ley Orgánica (LOMCE) 8/20I3, de 9 de diciembre, para la mejora de la educación. Boletín Oficial del Estado, n. ${ }^{\circ} 295$. Recuperado el 23 de noviembre de 2020, de https://www.boe.es/eli/es/lo/2013/ı2/o9/8/con.

Lieberman, G. A. y Hoody, L. (1998). Closing the Achievement Gap: Using the Environment as an Integrating Context for Learning. School, K-I2. 64. USA: State Education and Environment Roundtable. Recuperado el 23 de noviembre de 2020, de http://www.seer.org/extras/ execsum.pdf.

Miklitz, I. (2000). Der Waldkindergarten. Dimensionen eines pädagogischen Ansatzes. Neuwied, Berlin: Luchterhand. 
BOSQUESCUELA.

UN MODELO DE ESCUELA SOSTENIBLE EN LA NATURALEZA PHILIP BRUCHNER Y ANTONIO ARAGÓN REBOLLO

Ministerio de Educación, Cultura y Deporte. (29 de enero de 20i5). Orden ecd/65/20i5, de 2i de enero, por la que se describen las relaciones entre las competencias, los contenidos y los criterios de evaluación de la educación primaria, la educación secundaria obligatoria y el bachillerato. Boletín Oficial del Estado, n. ${ }^{\circ} 25,2015$. Recuperado el 23 de noviembre de 2020, de https://www.boe.es/buscar/doc.php?id=BOE-A-20I5-738.

Ministerio de Medio Ambiente. (1999). Libro Blanco de la Educación Ambiental en España. Madrid: Ministerio de Medio Ambiente - Centro de Publicaciones.

Muela, A.; Larrea, I.; Miranda, N. y Barandiaran, A. (20i9). Improving the quality of preschool outdoor environments: getting children involved. European Early Childhood Education Research Journal, 27:3, 385-396. DoI: 10.1080/1350293X.2019.1600808

Nisbet, E. K.; Zelenski, J. M. y Murphy, S. A. (20II). Happiness is in our Nature: Exploring Nature Relatedness as a Contributor to Subjective Well-Being. Journal of Happiness Studies, $I 2(2), 303-322$.

O'BRIEN, L. (2009). Learning outdoors: the Forest School approach. Education, 3-13, 37:I, 45-60. DOI:IO.1080/03004270802291798

Organización Naciones Unidas (Onu). (20I5a). Objetivos de Desarrollo sostenible. Recuperado el 23 de noviembre de 2020 , de https://www.un.org/sustainabledevelopment/es/objetivos-de-desarrollo-sostenible/.

Organización Naciones Unidas (ONu). (20I5b). Objetivo 4: Garantizar una educación inclusive, equitativa y de calidad y promover oportunidades de aprendizaje durante toda la vida para todos. Recuperado el 23 de noviembre de 2020 , de https://www.un.org/sustainabledevelopment/es/education/.

Radiotelevisión Española (RTVe). (2OI2). El proyecto Bosquescuela. En La Aventura del Saber. Recuperado el 23 de noviembre de 2020 , de https://www.youtube.com/watch?v=R9nusANoAqM.

Radiotelevisión Española (RTVe). (20I5). Philip Bruchner, Silvia Corchero y Ana Palomino. Bosquescuela. En La Aventura del Saber. Recuperado el 23 de noviembre de 2020, de https://www.rtve.es/alacarta/videos/la-aventura-del-saber/aventurabosqueescuela/2988782/.

Radiotelevisión Española (RTVE). (20I6). Madrid: RTVE. Recuperado el 23 de noviembre de 2020, de https://www.youtube.com/watch?v=A2UIUJAvUCQ.

Rodríguez, H.; Aragón, T. y Moya, J. (2020). Proyecto Ciclos: Un nuevo currículo en la práctica educativa. En J. Moya y A. Zubillaga Del Río, Un currículo para un mundo sostenible \#ODS2030. Madrid: Anaya.

Rosenberg, M. (2006). Comunicación No Violenta, un Lenguaje de Vida. Buenos Aires: Gran Aldea Ediciones.

SANDSETER, E. B. H. (2007). Categorizing risky play - How can we identify risk-taking in children's play? European Early Childhood Education Research Journal, I5(2), 237-252.

Schaffert, S. (2004). Der Waldkindergarten. Recuperado el 23 de noviembre de 2020, de https://www.kindergartenpaedagogik.de/fachartikel/paedagogische-ansaetze/moderne-paedagogische-ansaetze/ı216.

Skaugen, R. y Fiskum, T. A. (20I5). How Schools with Good Academic Results Justify Their Use of Outdoor Education. International Education Research, 3(4), 16-31. http://doi. org/IO.I2735/ier.v3i4pi6

Soga, M.; Gaston, K. J.; Yamaura, Y.; Kurisu, K. y Hanaki, K. (20i6). Both Direct and Vicarious Experiences of Nature Affect Children's Willingness to Conserve Biodiversity. International Journal of Environmental Research and Public Health, ${ }_{3} 3(6), 529$. https://doi. org/Io.3390/ijerphi3060529

STREelasky, J. (20I9). A forest-based environment as a site of literacy and meaning making for kindergarten children. Literacy, 53, 95-IOI. https://doi.org/IO.IIII/lit.I2I55 
BOSQUESCUELA.

UN MODELO DE ESCUELA SOSTENIBLE EN LA NATURALEZA PHILIP BRUCHNER Y ANTONIO ARAGÓN REBOLLO

Ward, C.; Aspinali, P. y Montarzino, A. (2007). The Childhood Factor: Adult Visits to Green Places and the Significance of Childhood Experience. Environ. Behav, 40, III-I43. DOI:I0.1177/0013916507300II9

Wells, N. M. y Evans, G. W. (2003). Nearby nature: a buffer of life stress among rural children. Environ. Behav., 35, 3II-330. https://doi.org/Io.II77/001391650303500300I

Wells, N. M. y Lekies, K. S. (2006). Nature and the Life Course: Pathways from Childhood Nature Experiences to Adult Environmentalism. Children, Youth and Environments, I6, I-24.

Wistoft, K. y Dyg, P. M. (20I8). Wellbeing in school gardens: the case of the Gardens for Bellies food and environmental education program. Environmental Education Research, 24(8), II77-II9I. https://doi.org/IO.IO80/13504622.20I8.I434869

\section{Anexo}

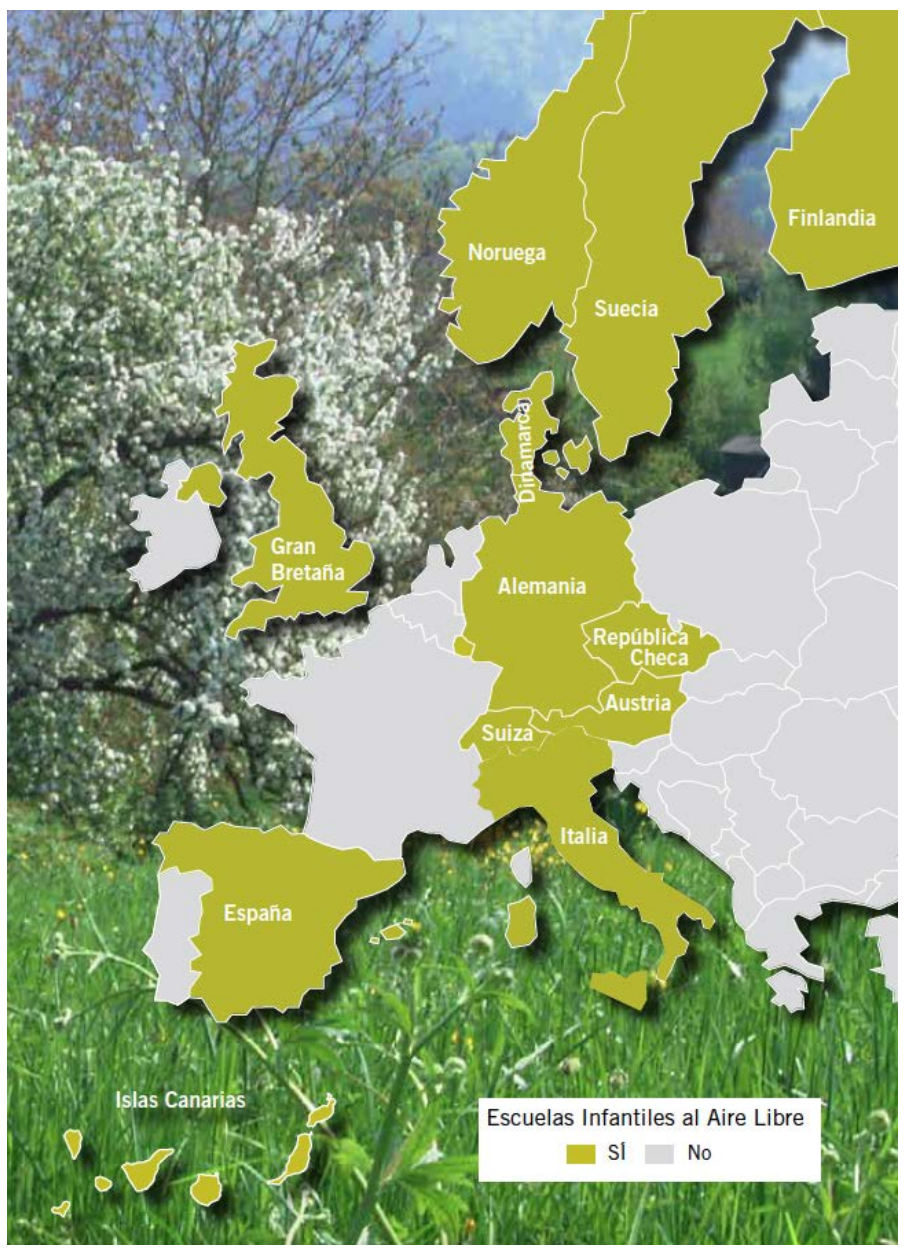

FIGURA I 
BOSQUESCUELA.

UN MODELO DE ESCUELA SOSTENIBLE EN LA NATURALEZA

PHILIP BRUCHNER Y ANTONIO ARAGÓN REBOLLO

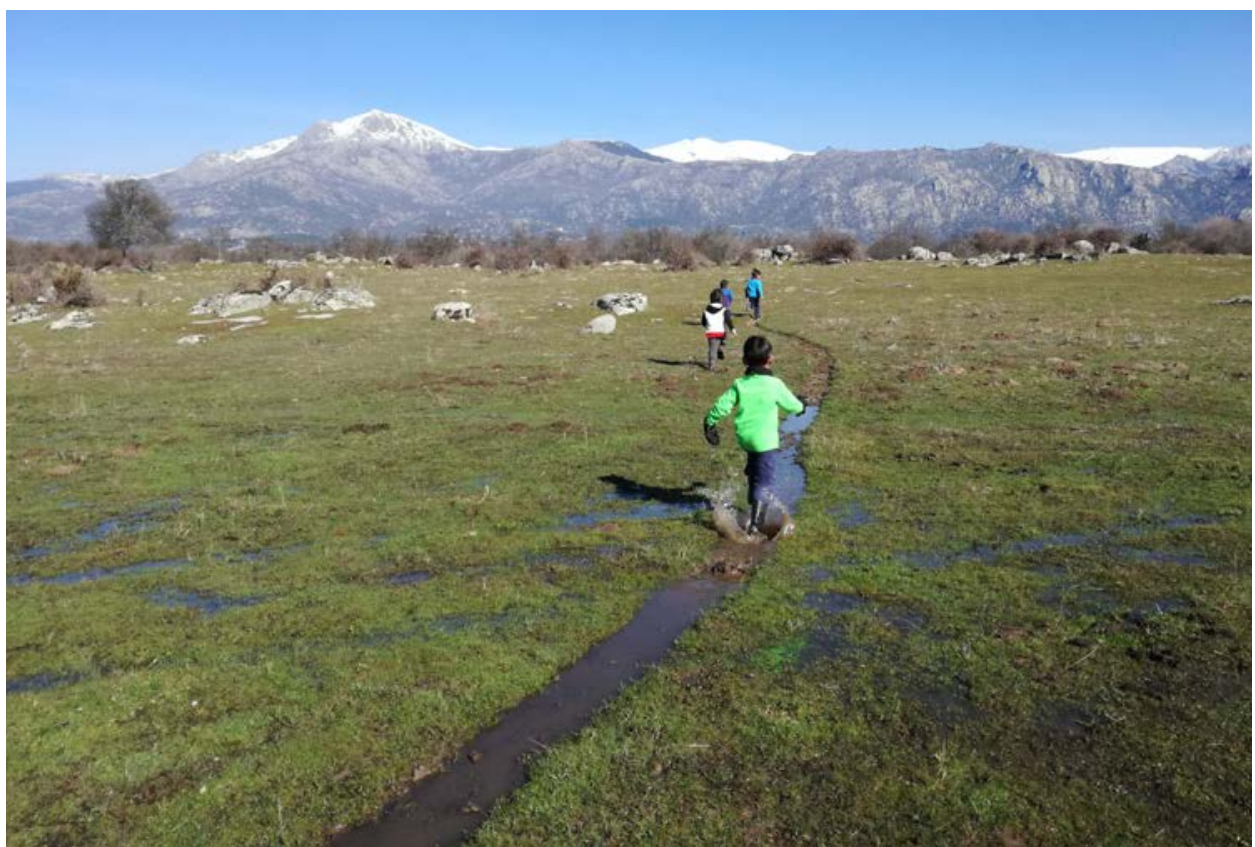

FiguRA 2

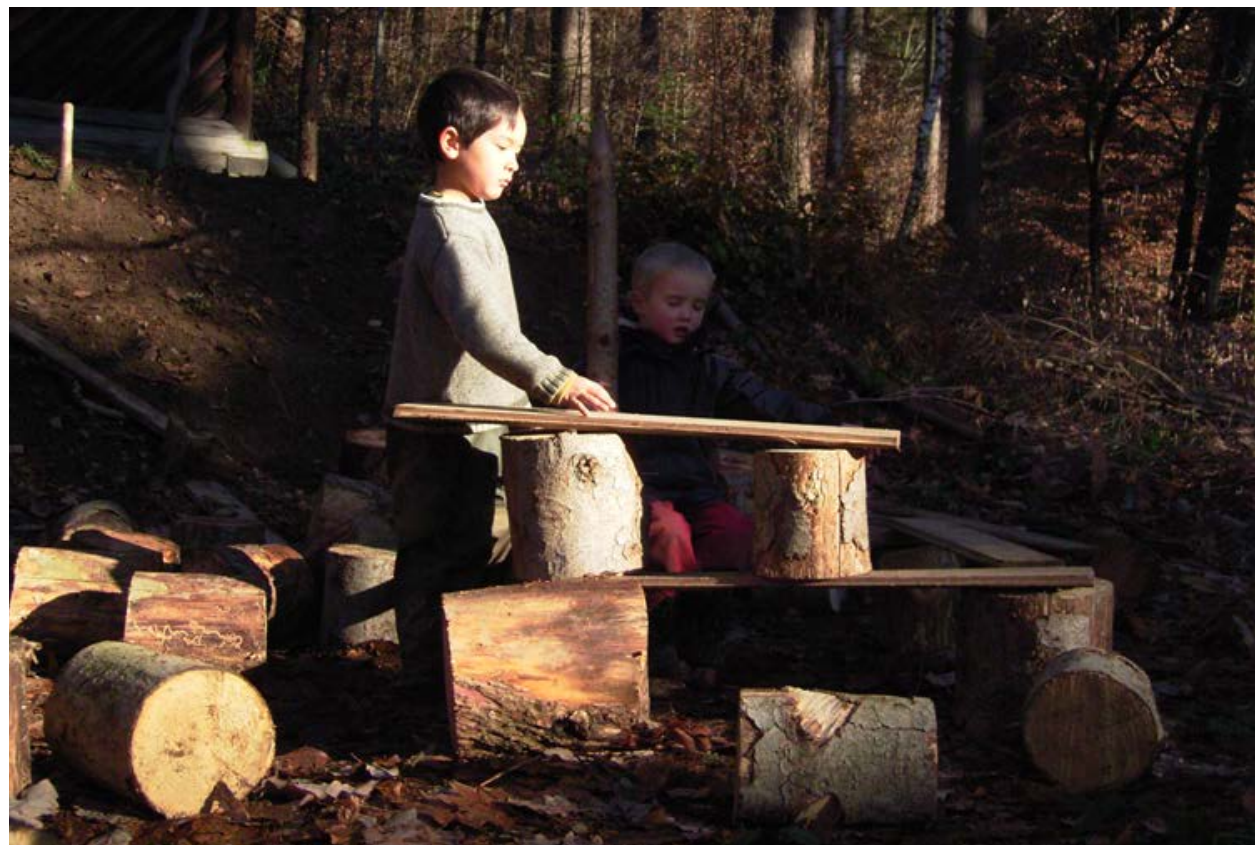

FIGURA 3

Ediciones Universidad de Salamanca / @080

Aula, 27, 202 I, pp. 209-233 
BOSQUESCUELA.

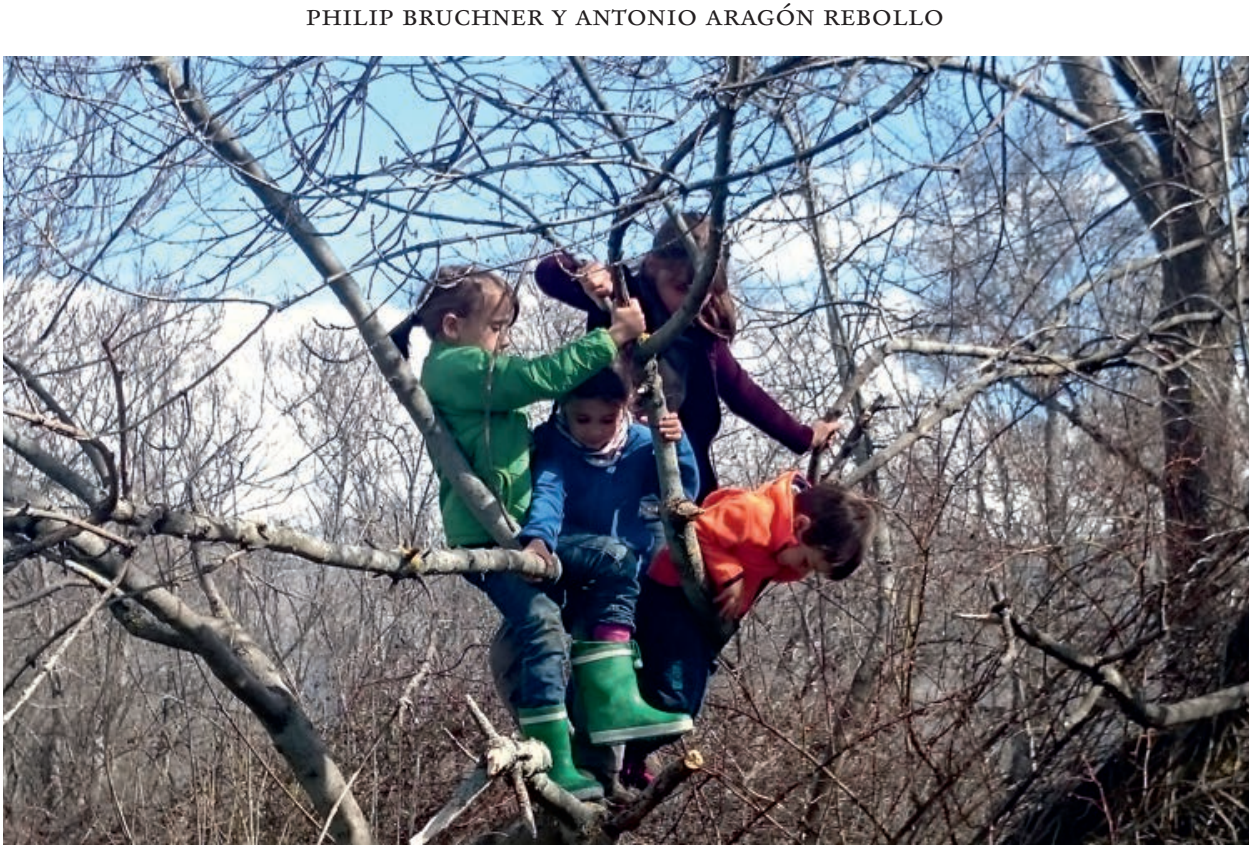

FIGURA 4

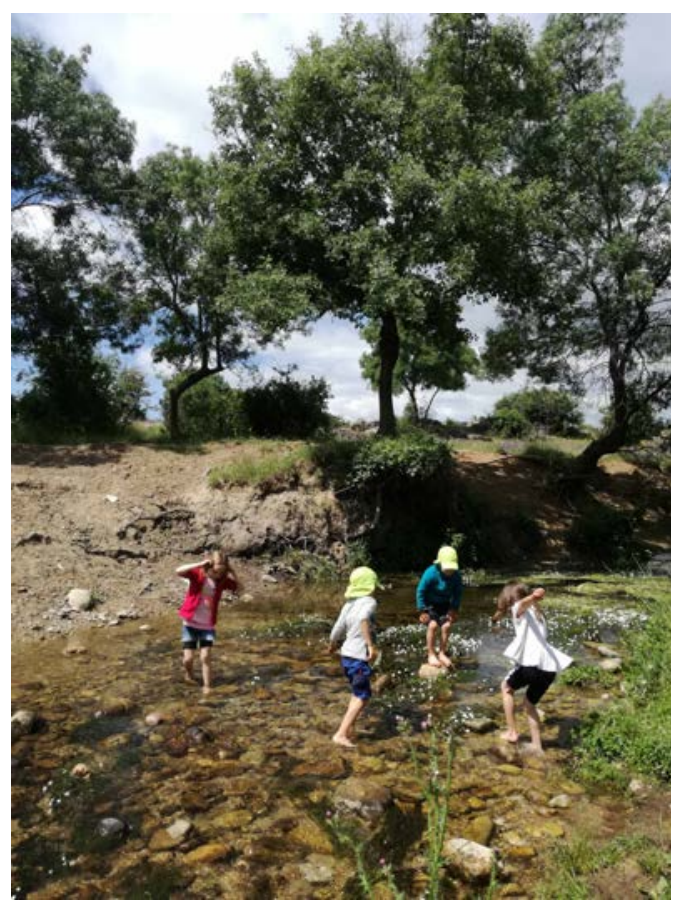

Figura 5 
BOSQUESCUELA.

UN MODELO DE ESCUELA SOSTENIBLE EN LA NATURALEZA

PHILIP BRUCHNER Y ANTONIO ARAGÓN REBOLLO

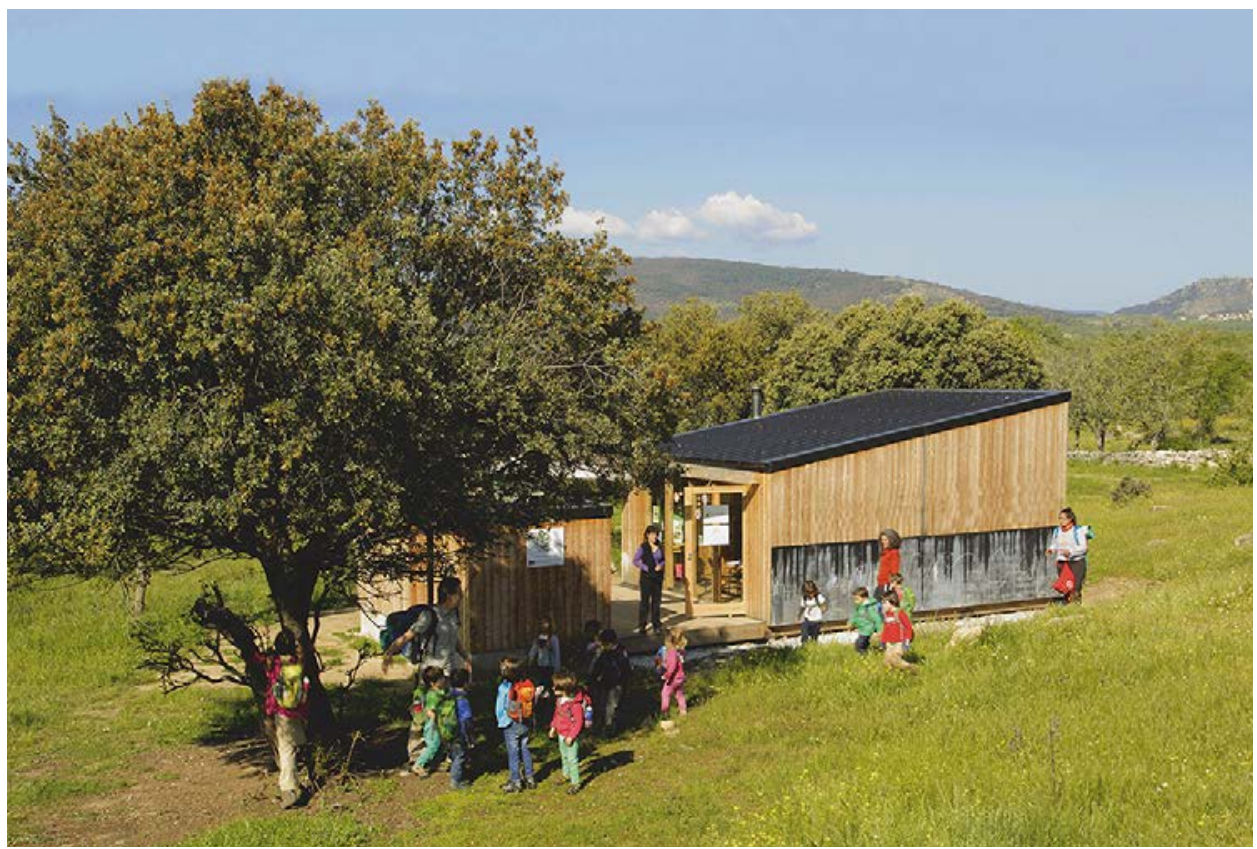

FiguRA 6

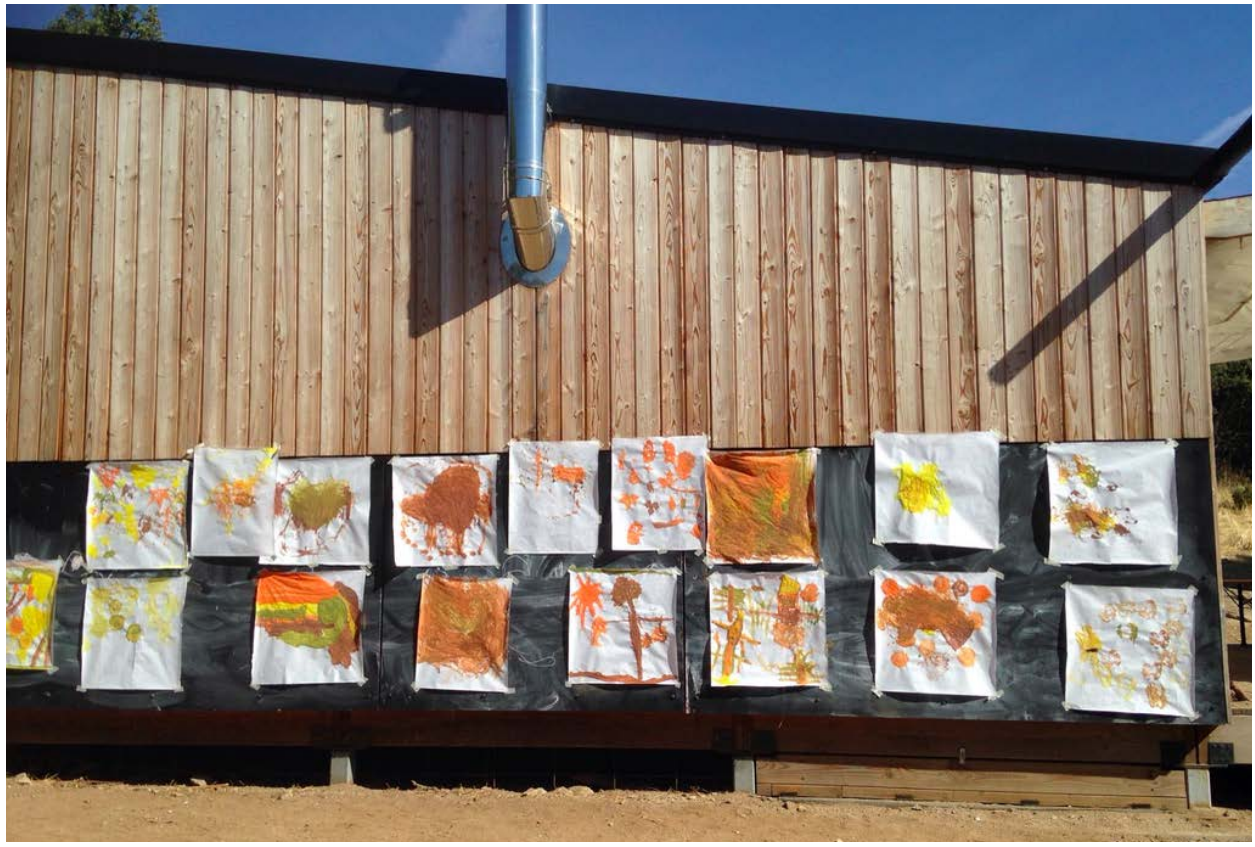

FigURA 7

Ediciones Universidad de Salamanca / @(1)

Aula, 27, 202 I, pp. 209-233 


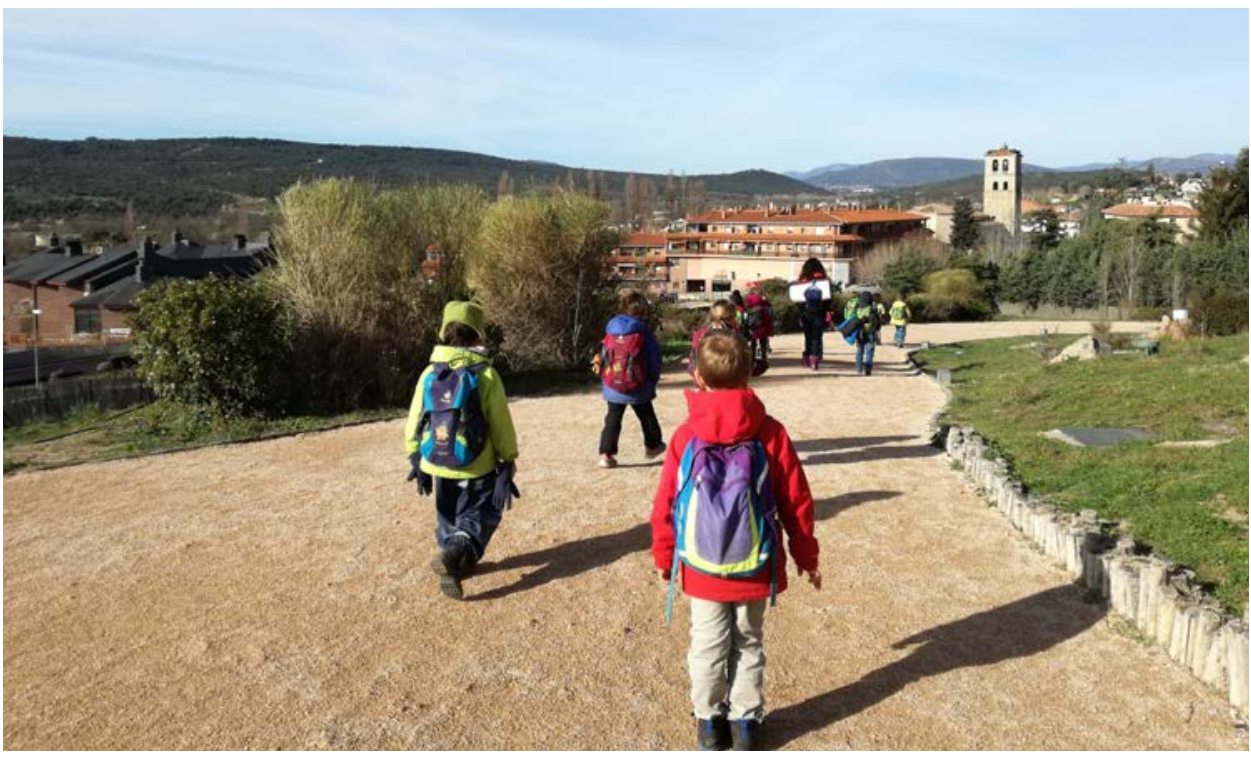

FIGURA 8

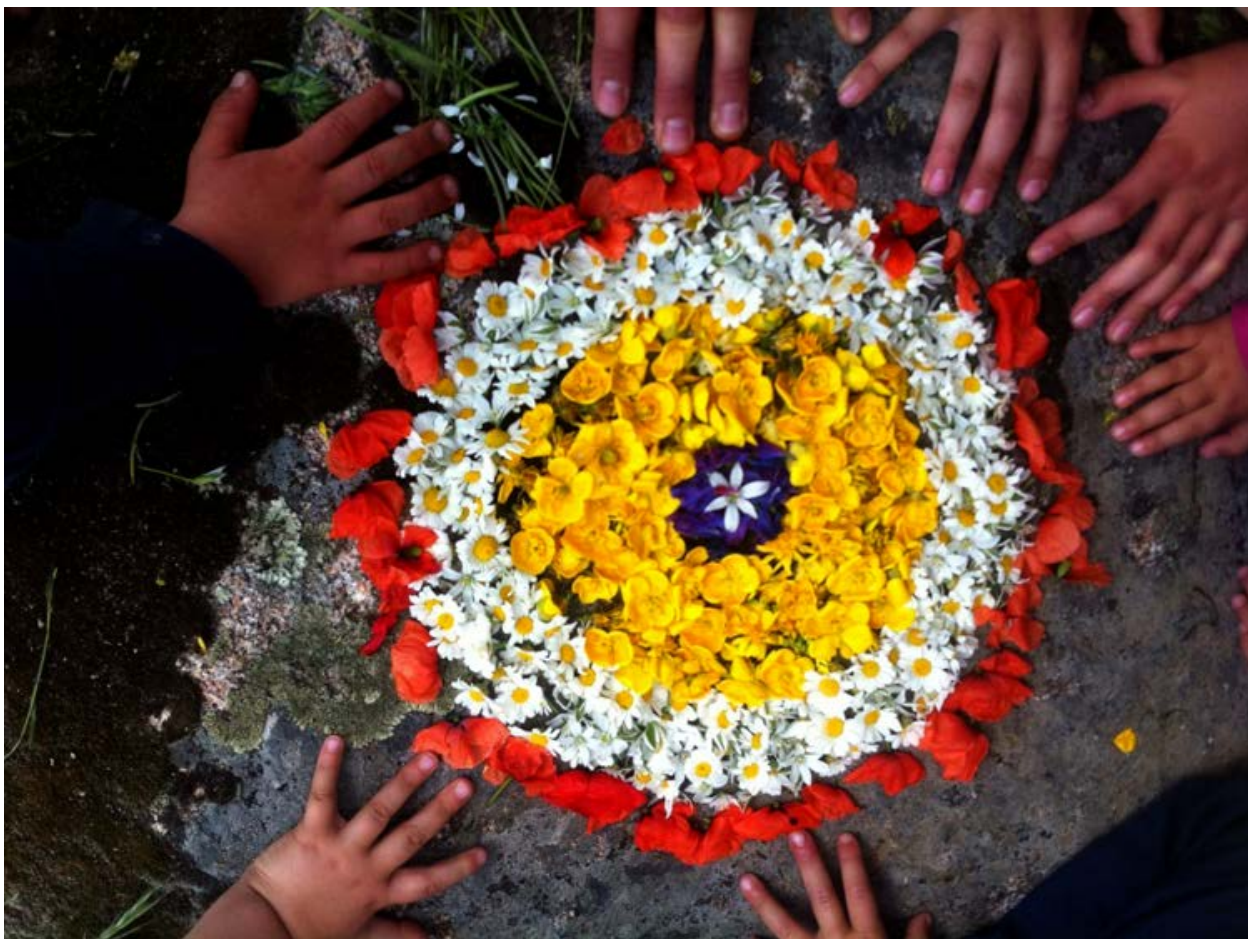

FigURA 9 
BOSQUESCUELA.

UN MODELO DE ESCUELA SOSTENIBLE EN LA NATURALEZA

PHILIP BRUCHNER Y ANTONIO ARAGÓN REBOLLO

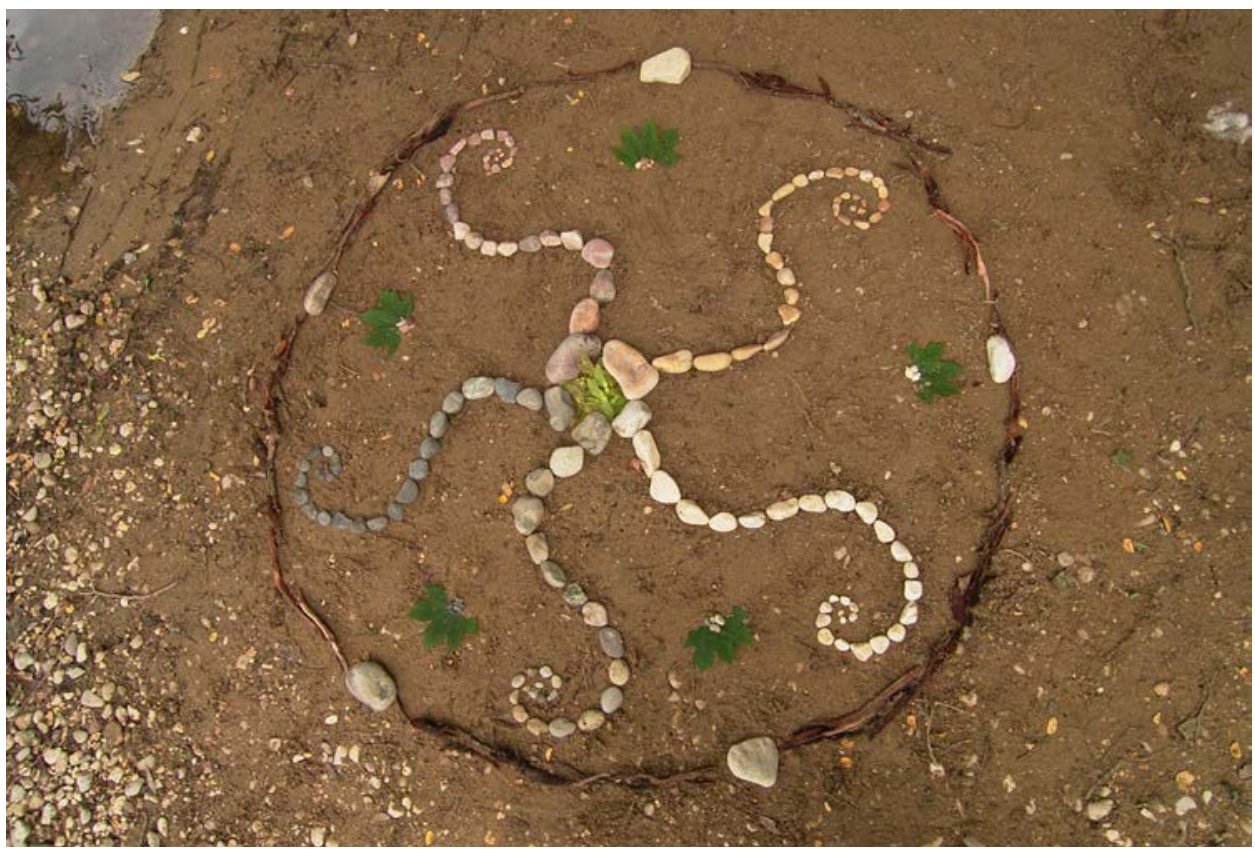

Figura IO

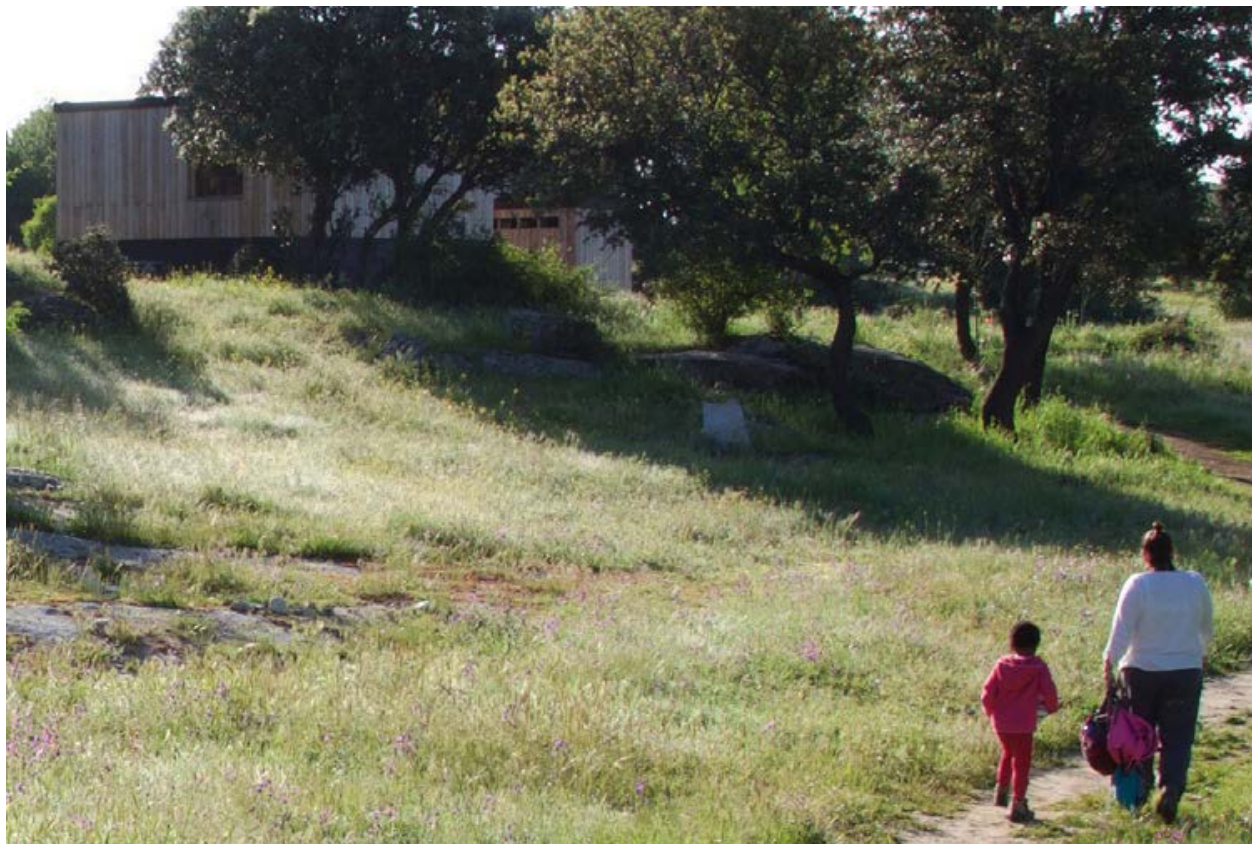

FIGURA II

Ediciones Universidad de Salamanca / @Q80

Aula, 27, 202 I, pp. 209-233 
BOSQUESCUELA.

30

UN MODELO DE ESCUELA SOSTENIBLE EN LA NATURALEZA

PHILIP BRUCHNER Y ANTONIO ARAGÓN REBOLLO

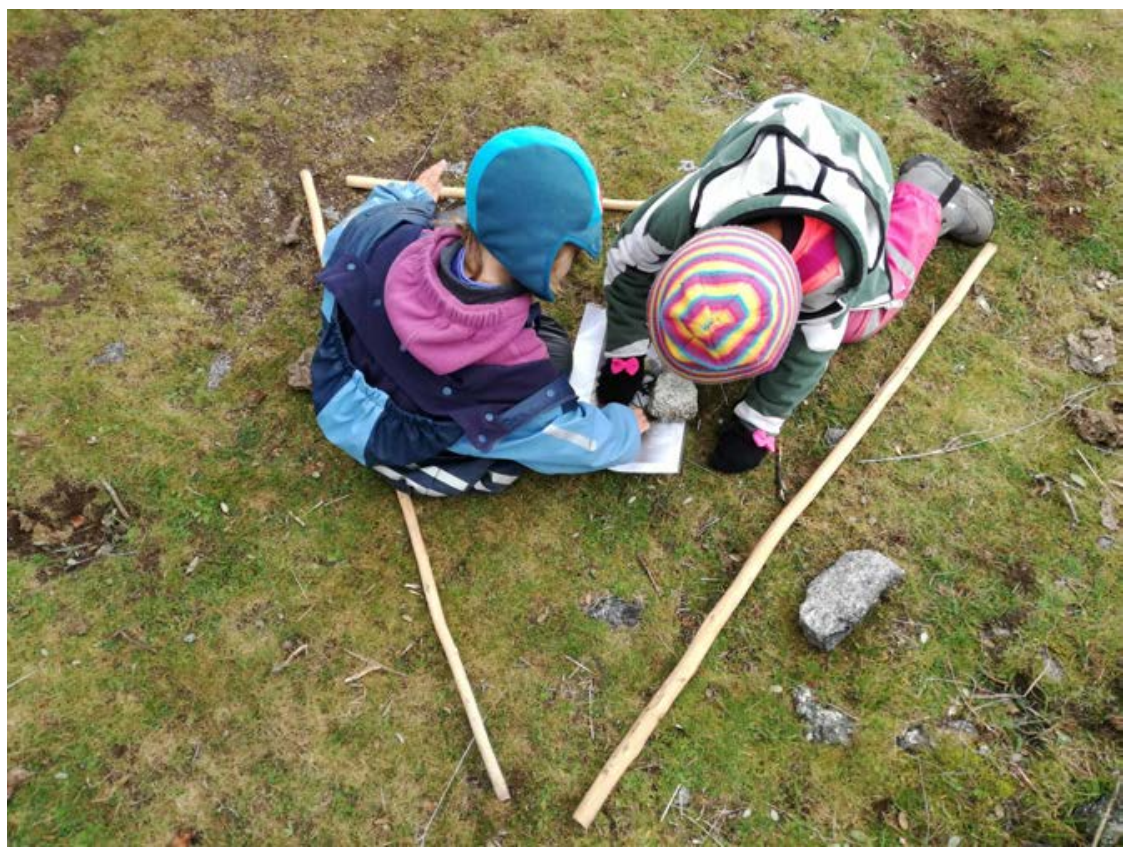

FigURA I2

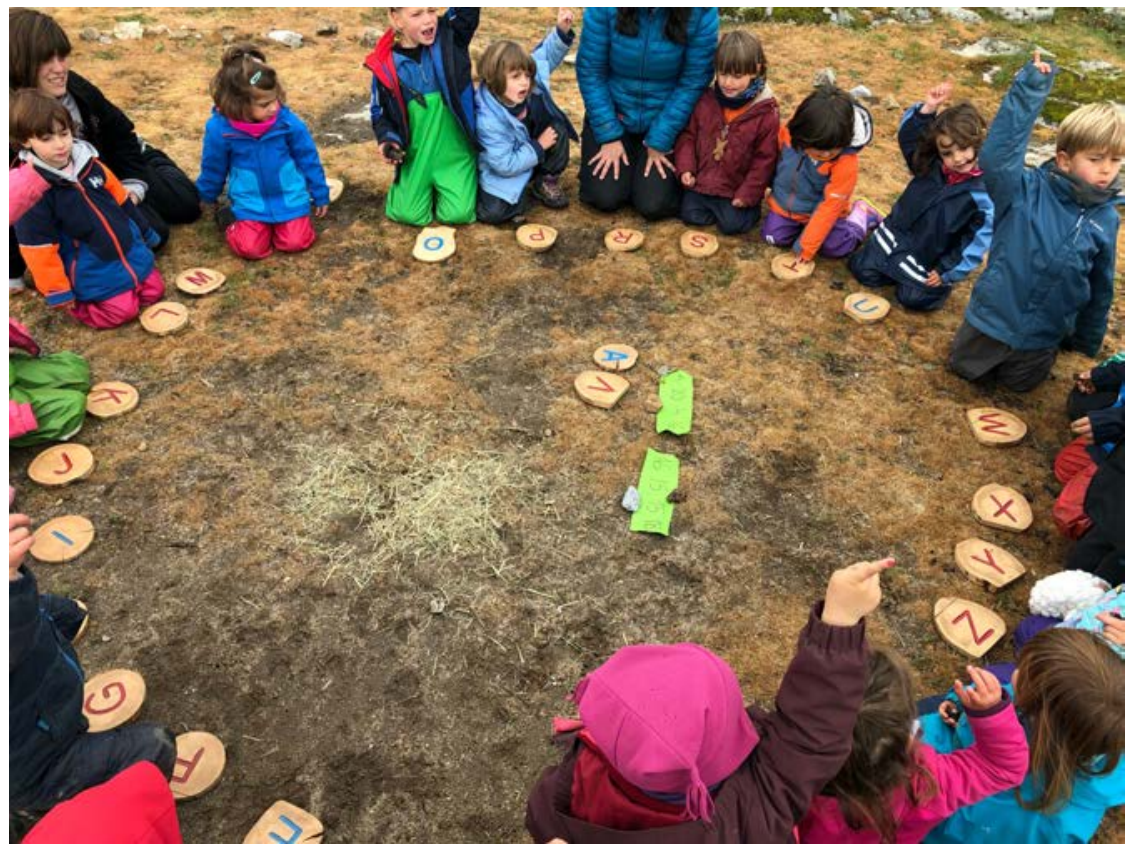

FIgURA I3

Ediciones Universidad de Salamanca / @Q80

Aula, 27, 2021, pp. 209-233 
BOSQUESCUELA.

UN MODELO DE ESCUELA SOSTENIBLE EN LA NATURALEZA

PHILIP BRUCHNER Y ANTONIO ARAGÓN REBOLLO

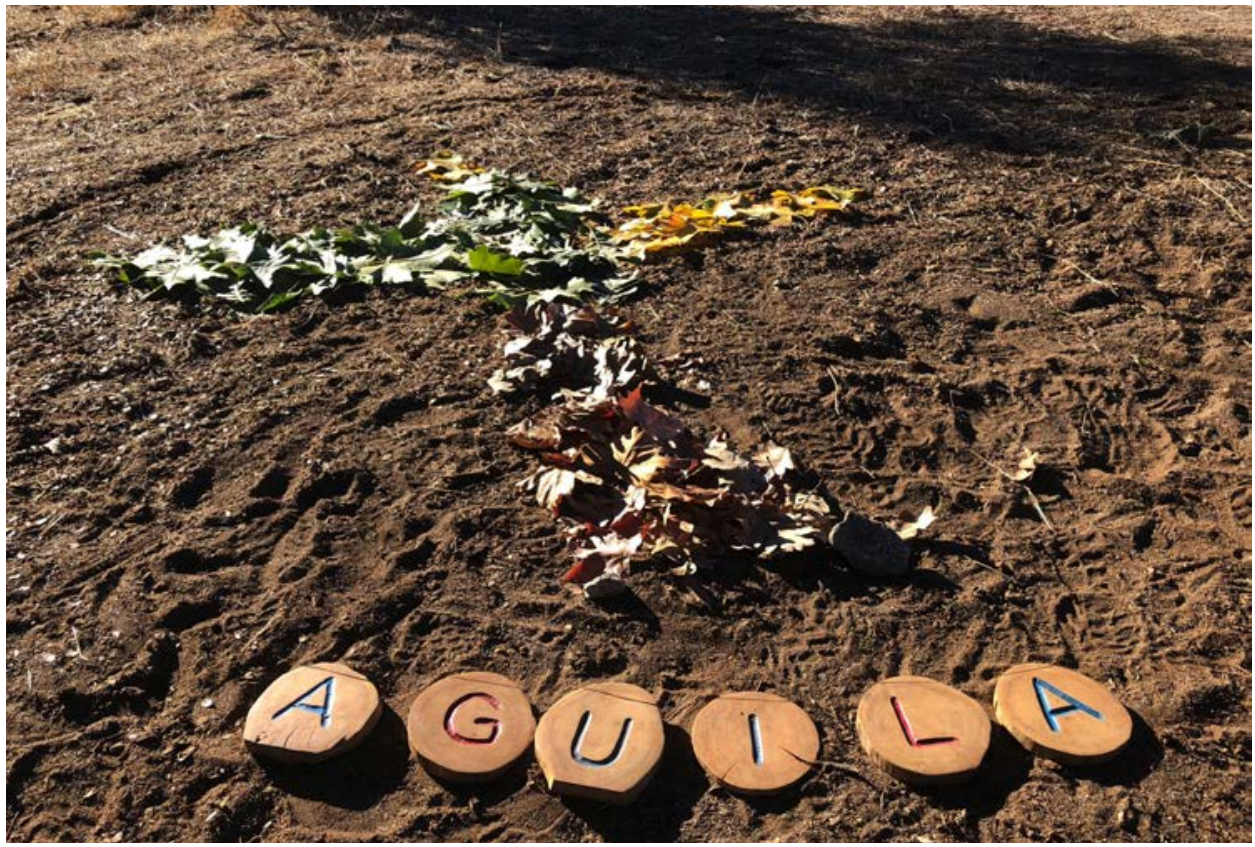

FIGURA I4

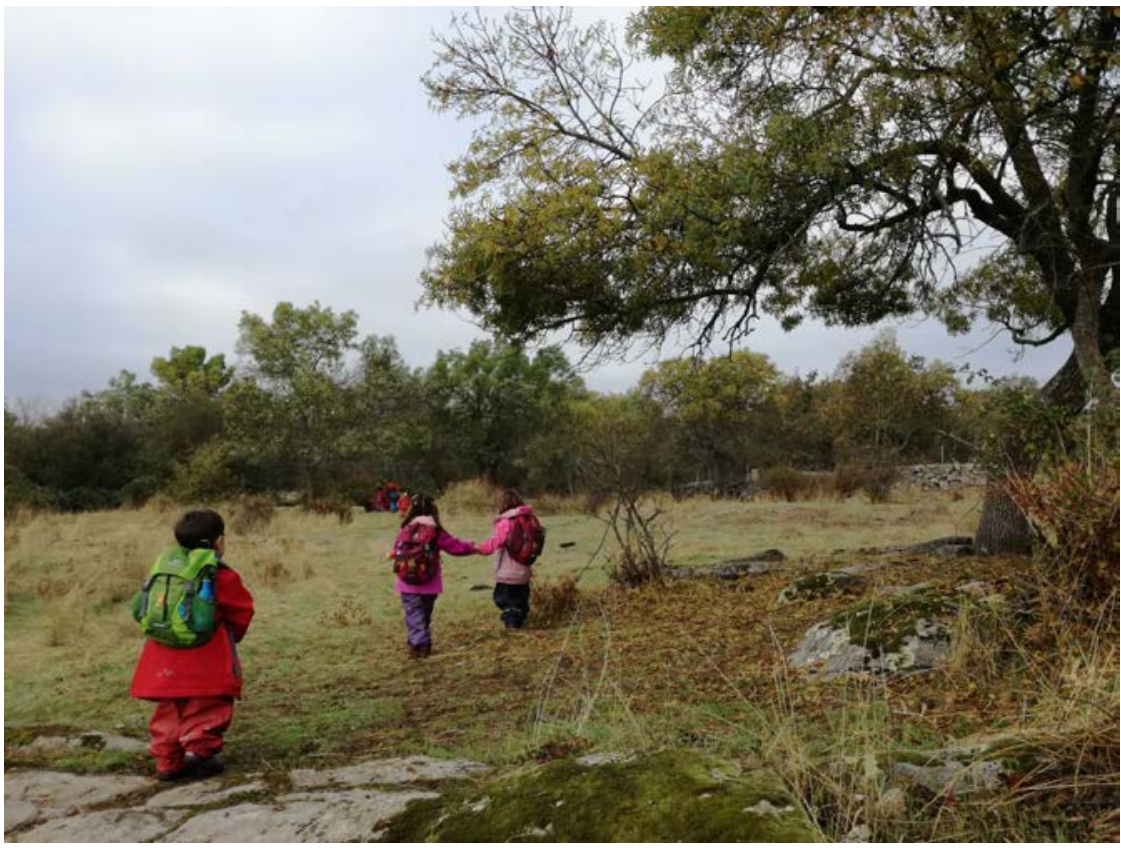

FIgURA I5 
BOSQUESCUELA.

UN MODELO DE ESCUELA SOSTENIBLE EN LA NATURALEZA

PHILIP BRUCHNER Y ANTONIO ARAGÓN REBOLLO

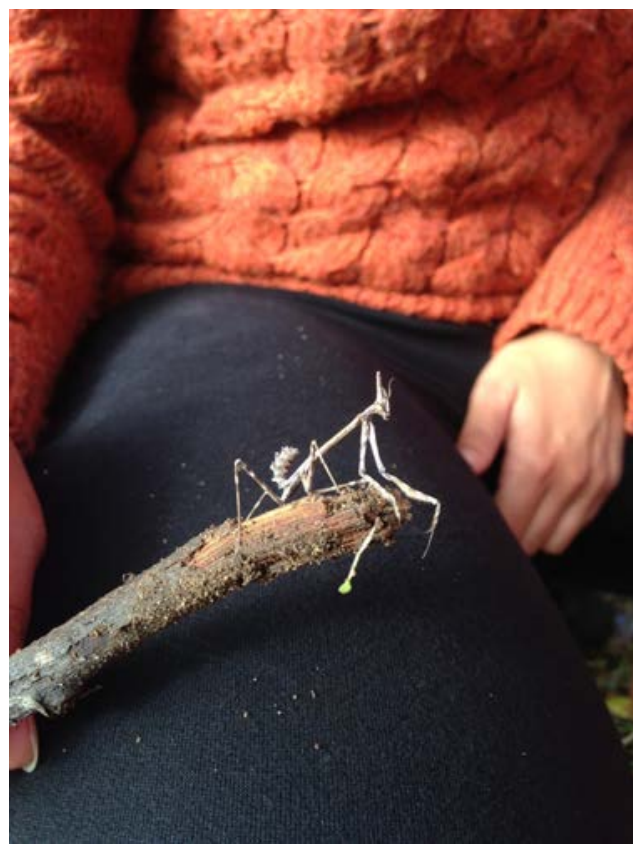

FiguRA I6

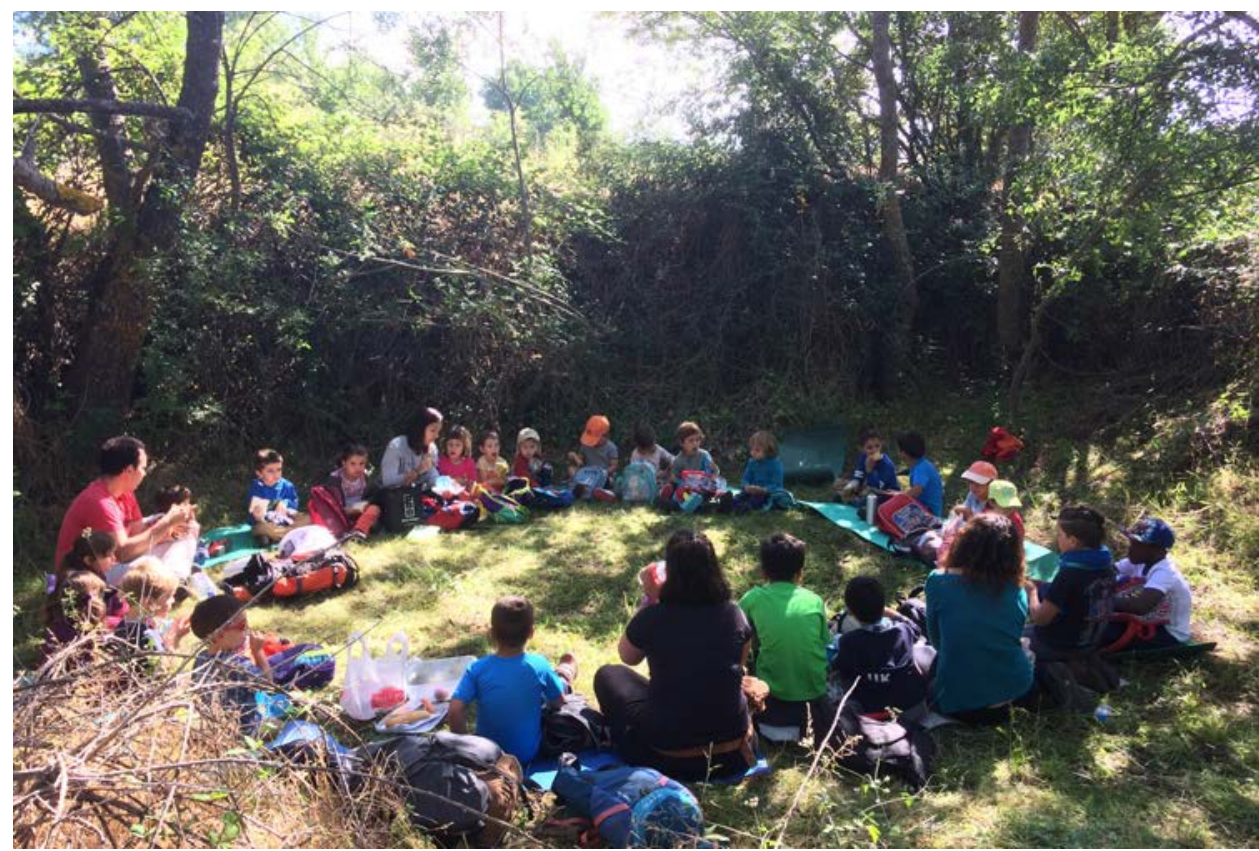

FigURA I7

Ediciones Universidad de Salamanca / @0 (100

Aula, 27, 2021, pp. 209-233 
BOSQUESCUELA.

UN MODELO DE ESCUELA SOSTENIBLE EN LA NATURALEZA

PHILIP BRUCHNER Y ANTONIO ARAGÓN REBOLLO

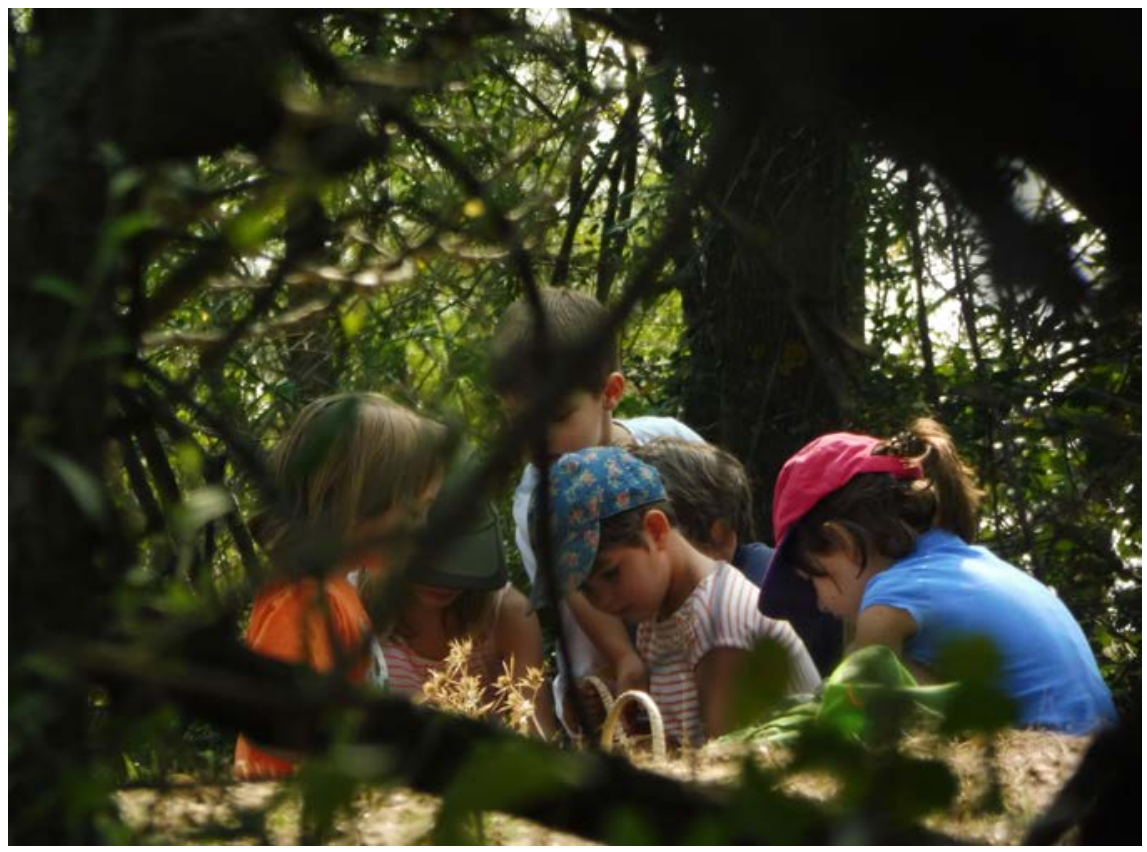

FIGURA I8

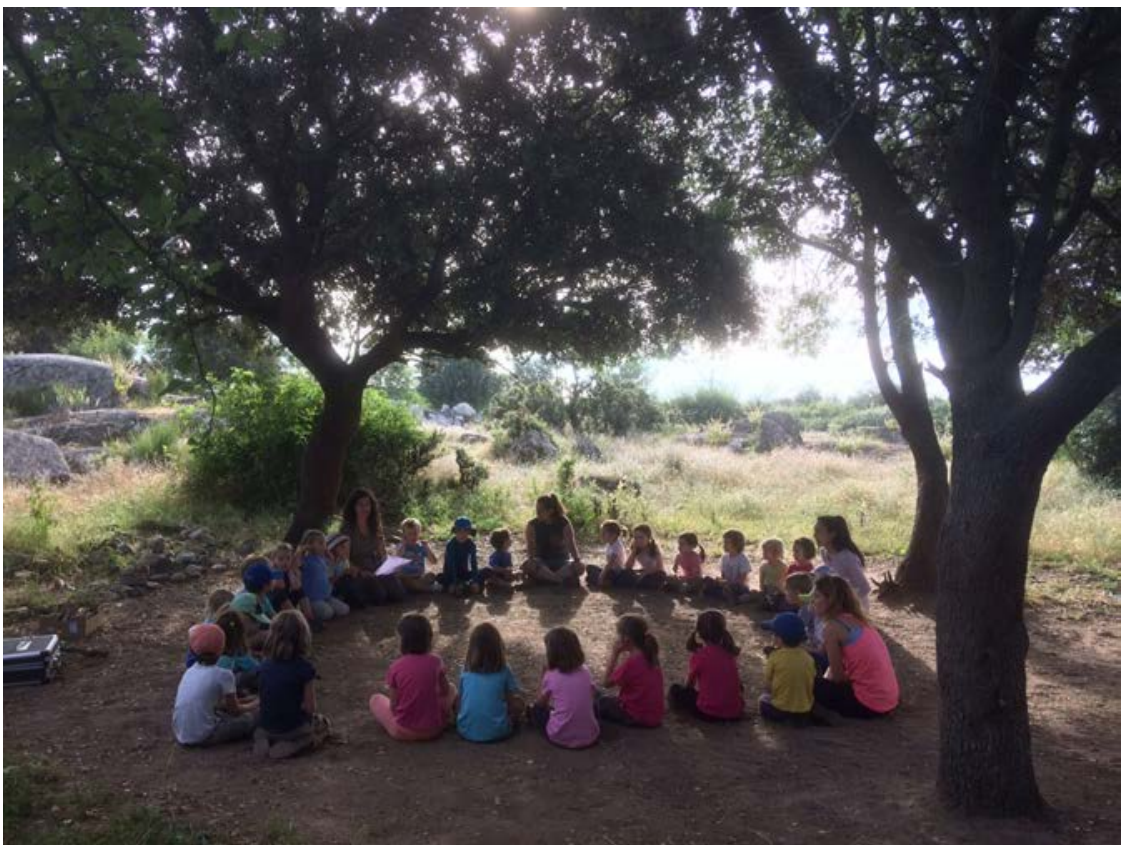

FIgURA I9 
